TRANSACTIONS OF THE

AMERICAN MATHEMATICAL SOCIETY

Volume 356, Number 9, Pages 3483-3504

S 0002-9947(03)03351-8

Article electronically published on October 28, 2003

\title{
DEFINABILITY IN THE LATTICE OF EQUATIONAL THEORIES OF COMMUTATIVE SEMIGROUPS
}

\author{
ANDRZEJ KISIELEWICZ \\ To Professor Ralph McKenzie
}

\begin{abstract}
In this paper we study first-order definability in the lattice of equational theories of commutative semigroups. In a series of papers, J. Ježek, solving problems posed by A. Tarski and R. McKenzie, has proved, in particular, that each equational theory is first-order definable in the lattice of equational theories of a given type, up to automorphism, and that such lattices have no automorphisms besides the obvious syntactically defined ones (with exceptions for special unary types). He has proved also that the most important classes of theories of a given type are so definable. In a later paper, Ježek and McKenzie have "almost proved" the same facts for the lattice of equational theories of semigroups. There were good reasons to believe that the same can be proved for the lattice of equational theories of commutative semigroups. In this paper, however, we show that the case of commutative semigroups is different.
\end{abstract}

\section{InTRODUCTION}

In [17, 14, A. Tarski and R. McKenzie raised problems of first-order definability in a lattice $L$ of equational theories. A subset $S$ of $L$ is first-order definable in $L$ if there exists a formula $\Phi(T)$ in the first-order language of $L$ such that $\Phi(T)$ holds if and only if $T \in S$. An equational theory $T \in L$ is definable if the set $\{T\}$ is definable. Accordingly, we speak about definable relations and properties of the theories in $L$.

The language in question can be thought as one whose only nonlogical symbol is that of inclusion (or equivalently, the symbols of meet and join). It seems relatively poor, but the lattices of equational theories considered are usually large, and many interesting sets, from the point of view of equational logic, can be defined in this language. For general results see [14, 6, 7, 8, 9, and for some results on equational theories of rings and groups see [4, 5, 18,

In [10, Ježek and McKenzie studied first-order definability in the lattice of equational theories of semigroups. They proved that such sets as the set of finitely axiomatizable theories, the set of locally finite theories, and the set of theories of finite semigroups are all definable. Also they proved that each individual finitely axiomatizable locally finite theory is definable up to duality (i.e., up to inverting

Received by the editors June 14, 2002 and, in revised form, March 21, 2003.

2000 Mathematics Subject Classification. Primary 03C07; Secondary 03C05, 08B15, 20 M07.

This research was done while the author was a Fulbright Visiting Scholar at Vanderbilt University. Supported in part by Polish KBN grant P03A 00916. 
the order of occurrences of letters in words). They have not been able to finish their program that would lead to the conclusion that "locally finite" in the last statement may be dropped, and that, in consequence, the lattice of equational theories of semigroups has no nontrivial automorphism except for the duality. These questions still remain open.

During my visit at Vanderbilt University, Ralph McKenzie asked me if my description in [1] can be used to answer these kinds of questions for the lattice $\mathcal{L}(\mathrm{Com})$ of equational theories of commutative semigroups.

In [1], I described equational theories of commutative semigroups in terms of certain order filters, integer parameters, and the so-called remainders. While the filters and integer parameters lead to a very regular, distributive sublattice of $\mathcal{L}(\mathrm{Com})$ that is easy to handle, the remainders introduce interferences that make the whole lattice quite complex (for example, all finite lattices are contained in $\mathcal{L}(\mathrm{Com})$ as sublattices). The remainders are equivalence relations on sequences of positive integers satisfying a few technical conditions. What is however the most important is that they have a finite character, and are relatively small parts of theories. Algorithms and formulas in [11] provide a general method of computing in equational theories and varieties of commutative semigroups. This method has already proved to be useful in solving a number of problems [3, 1, 2, 12, 13. In this paper we use it to solve the problem suggested by McKenzie.

From the results of [10] it follows that each equational theory of commutative semigroups is definable in the lattice of equational theories of all semigroups, since each such (nontrivial) theory is finitely axiomatizable and locally finite. This does not imply that each such theory is definable in $\mathcal{L}(\mathrm{Com})$, because the universe of $\mathcal{L}(\mathrm{Com})$ is much smaller. However, this lattice seems rich and complicated enough to make such a statement plausible. In particular, the facts that, on the one hand, $\mathcal{L}(\mathrm{Com})$ contains all finite lattices and, on the other hand, each theory in $\mathcal{L}(\mathrm{Com})$ is finitely axiomatizable, made us conjecture that all the theories in $\mathcal{L}(\mathrm{Com})$ are definable and that $\mathcal{L}(\mathrm{Com})$ itself has no nontrivial automorphism.

Although no result of [10] can be applied directly to our problem, we were trying to follow the approach used in [10, (and earlier in [6, 7, 8]) as long as possible. The main idea of this approach is to encode equations (and sequences of words) in the ordered set of patterns. (A pattern is an orbit under the action of automorphisms on the free semigroup; and the order relation on patterns is inherited from the relation "substituble into" between words.) Now, the ordered set of patterns is order isomorphic to the set of the so-called ideal theories, which is definable. As a result, the ideal theories can be used like Gödel numbers to encode syntactical properties.

In the case of $\mathcal{L}(\mathrm{Com})$, this must be taken modulo commutativity. The ideal theories correspond to certain Schwabauer theories, and patterns correspond to sets of positive integers (unordered $n$-tuples). Unfortunately, equations do not correspond to pairs of patterns, since possible permutations of variables in words can no longer be encoded within the patterns. At this point we expected that the tools of [11] would prove to be useful.

There were two surprises while performing this program. First, unlike in [10], the set of ideal theories turned out to be too poor to define much structure in $\mathcal{L}(\mathrm{Com})$. Yet, using [11, we were able to prove that the larger set of so-called Schwabauer theories has all the nice properties of ideal theories. Schwabauer theories, known 
to form a maximal modular sublattice of $\mathcal{L}(\mathrm{Com})$, are definable both as a set and individually. This means, in particular, that the sublattice of Schwabauer theories remains fixed under any automorphism (which is one more reason to call this sublattice the "skeleton" of $\mathcal{L}(\mathrm{Com}))$. Using it, we can go further, to prove that many individual theories and interesting sets of theories are definable in $\mathcal{L}(\mathrm{Com})$. However, we have not been able to establish the definability of one-based theories generated by certain regular and quite simple equations-because this turned out not to be true. Instead, our final section is devoted to demonstrating that $\mathcal{L}(\mathrm{Com})$ admits a nontrivial automorphism.

\section{Preliminaries}

We assume that the reader has basic knowledge in the areas of first-order logic, equational logic and lattice theory. Some necessary details can be found in the introductions to [10] and [11. In this section we recall definitions and results from [11] that we use throughout this paper.

The crucial structure behind the equational theories of commutative semigroups is the set $\Gamma$ of all finite sequences $\left(\alpha_{1}, \ldots, \alpha_{n}\right)$ of nonnegative integers such that at least one $\alpha_{i} \neq 0$, along with some operations and an order relation described below.

For a sequence $\mathbf{a}=\left(\alpha_{1}, \ldots, \alpha_{n}\right) \in \Gamma$ and a permutation of the indices $f \in S_{n}$, we put $f(\mathbf{a})=\left(\alpha_{f(1)}, \ldots, \alpha_{f(n)}\right)$. In addition, for every $\gamma \geq 0$ we denote $h_{\gamma}(\mathbf{a})=$ $\left(\alpha_{1}, \ldots, \alpha_{n}, \gamma\right)$, and for all $1 \leq i, j \leq n, i \neq j$, we denote $g_{i j}(\mathbf{a})=\left(\alpha_{1}, \ldots, \alpha_{i}+\right.$ $\left.\alpha_{j}, \ldots, \alpha_{n}\right)$, where the latter is the sequence obtained from a by replacing $\alpha_{i}$ by $\alpha_{i}+\alpha_{j}$, and deleting $\alpha_{j}$.

For sequences $\mathbf{a}, \mathbf{b} \in \Gamma$, we define $\mathbf{a} \leq \mathbf{b}$ if and only if there exists an operation $f$ generated by the operations $g_{i j}, h_{1}, h_{0}$ and permutations such that $f(\mathbf{a})=\mathbf{b}$.

Clearly, $\leq$ is reflexive ( $f$ may be taken to be an identity permutation) and transitive, which means that $(\Gamma, \leq)$ is a quasi-ordered set. Further, note that two sequences are equivalent (the relation $\leq$ holds in both directions) if and only if they differ at most in the arrangement of elements and the number of zeros. Thus, in every equivalence class there is a unique nonincreasing sequence of positive integers determining the class, and $\leq$ can be viewed conveniently as the induced partial order on such sequences, treated as representatives of equivalence classes (cf. Figure 1).

It is proved in [11] (by means of another alternate definition of this order) that $(\Gamma, \leq)$ is actually a well-quasi-ordered set, which means, in particular, that there are no infinite descending chains in $\Gamma$ and every (order) filter $J$ in $\Gamma$ is finitely generated ([11, Proposition 1.5]). A filter $J$ generated by sequences $\mathbf{a}_{1}, \ldots, \mathbf{a}_{n}$ (i.e., the set of all sequences in $\Gamma$ greater or equal to one of $\left.\mathbf{a}_{1}, \ldots, \mathbf{a}_{n}\right)$ is denoted by $J=\left[\mathbf{a}_{1}, \ldots, \mathbf{a}_{n}\right]$. Obviously, each filter is a union of equivalence classes. The filters in $\Gamma$ correspond to certain theories of commutative semigroups as follows.

A commutative semigroup equation

$$
x_{1}^{\alpha_{1}} \ldots x_{n}^{\alpha_{n}}=x_{1}^{\beta_{1}} \ldots x_{n}^{\beta_{n}}
$$

will be identified with the pair $(\mathbf{a}, \mathbf{b})$ of the sequences $\mathbf{a}=\left(\alpha_{1}, \ldots, \alpha_{n}\right)$ and $\mathbf{b}=$ $\left(\beta_{1}, \ldots, \beta_{n}\right)$, and denoted briefly by $(\mathbf{a}, \mathbf{b})$. Usually we will assume that $\alpha_{i}+\beta_{i}>0$. An equation is nontrivial if $\alpha_{i} \neq \beta_{i}$ for some $i$.

An equational theory of commutative semigroups (in short, a theory) is a nonempty set of equations closed on taking logical consequences, which corresponds to applying the operations $g_{i j}, h_{1}, h_{0}$ and permutations to pairs $(\mathbf{a}, \mathbf{b})$, and using 


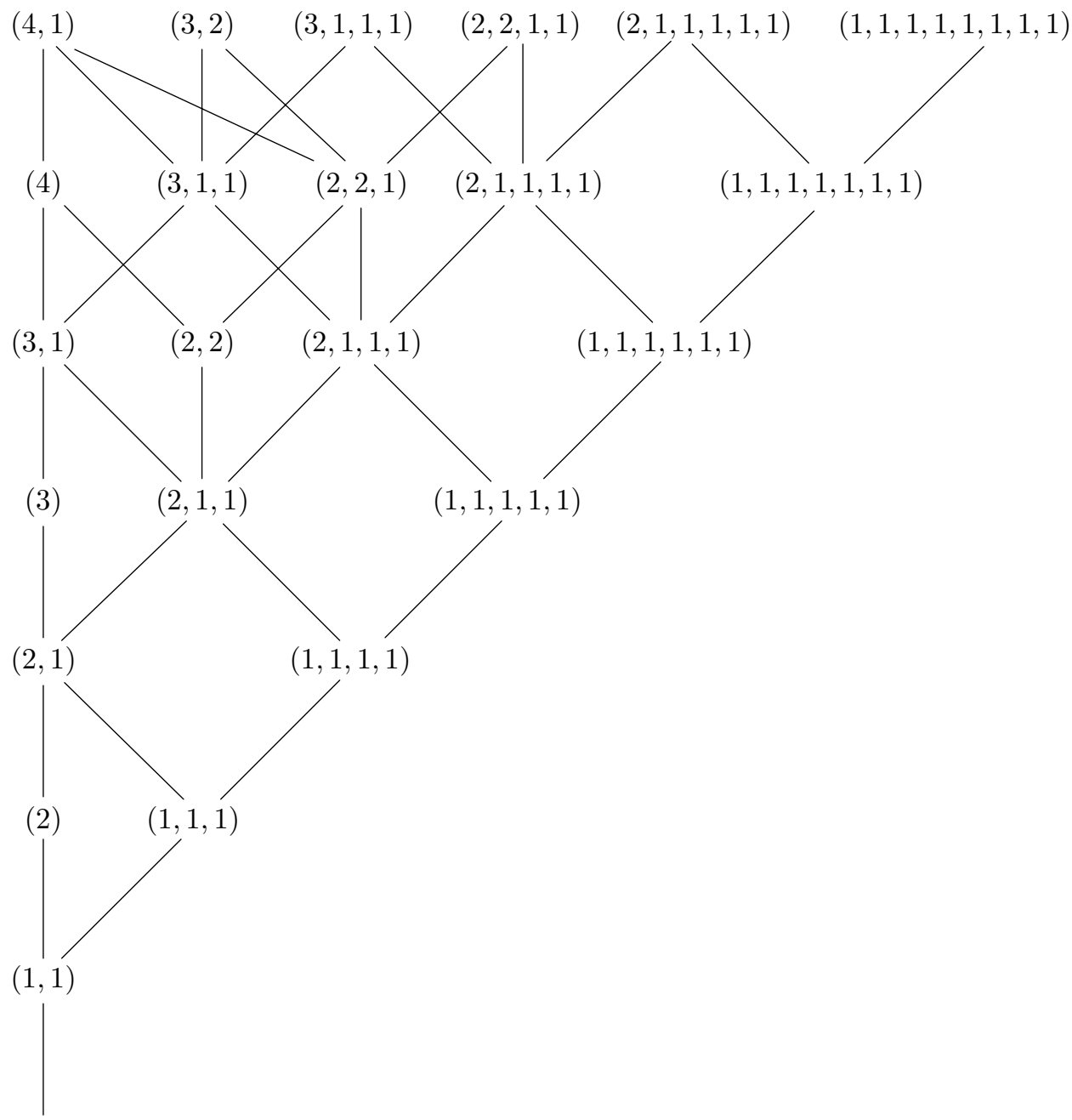

(1)

Figure 1. Ordered set of sequences up to equivalence.

transitivity (see [1] for more detail). The least theory consisting of the equations of the form $(\mathbf{a}, \mathbf{a})$ is called trivial.

Given a nonempty filter $J$ in $\Gamma$, define $E(J)$ to be the set of all equations $(\mathbf{a}, \mathbf{b})$ such that either both $\mathbf{a}, \mathbf{b} \in J$ or $\mathbf{a}=\mathbf{b}$. Then $E(J)$ is easily seen to be an equational theory (in [10, theories of this form are called ideal theories).

Further, for integers $m \geq 0$ and $r>0$ we define $E(J, m, r)$ to be the set of those pairs $(\mathbf{a}, \mathbf{b}) \in E(J)$ that satisfy the following two conditions:

$\left(C^{r}\right)$ for every $i, \alpha_{i} \equiv \beta_{i}(\bmod r)$,

$\left(C_{m}\right)$ for every $i$, if $\alpha_{i} \neq \beta_{i}$ then $\alpha_{i}, \beta_{i} \geq m$.

These conditions are invariant with regard to taking logical consequences. Therefore, each $E(J, m, r)$ is also an equational theory, and $E(J)=E(J, 0,1)$. The theories $E(J, m, r)$ are called Schwabauer theories or $S$-theories, in brief. 
Finally, we consider the following set of conditions for an equation $(\mathbf{a}, \mathbf{b})$, a filter $J$, and a set of equations $\pi$.

$(\pi 1)$ The conditions $\left(C^{r}, C_{m}\right)$ hold.

$(\pi 2)$ For every permutation $f \in S_{n},(f(\mathbf{a}), f(\mathbf{b})) \in \pi$.

$(\pi 3)$ For every $i$ with $\alpha_{i} \neq \beta_{i}$, and $\gamma=\min \left(\alpha_{i}, \beta_{i}\right)$, both $h_{\gamma}(\mathbf{a}), h_{\gamma}(\mathbf{b}) \in J$.

$(\pi 4)$ If $f$ is one of the operations $g_{i j}$ or $h_{1}$, then either $(f(\mathbf{a}), f(\mathbf{b})) \in \pi$ or both $f(\mathbf{a}), f(\mathbf{b}) \in J$.

Now, let $m \geq 0, r>0$ be integers and $J$ a nonempty filter contained in $[(m)]$ (the latter denotes the filter generated by one-element sequence $(m)$; for $m=0$ we define $[(0)]=\Gamma)$. Let $\pi$ be an equivalence relation on the set $\Gamma_{+} \backslash J$ of those finite sequences of positive integers that are not in $J$. If every block of $\pi$ contains sequences of one fixed length and for all pairs $(\mathbf{a}, \mathbf{b}) \in \pi$ the conditions $(\pi 1-\pi 4)$ above are satisfied, then $\pi$ is called a remainder of type $(J, m, r)$. For such a remainder we define $E(J, m, r, \pi)=E(J, m, r) \cup \pi$. Then, the main result in [11] (Theorem 4.8) can be stated as follows.

Theorem 2.1. Every set $E(J, m, r, \pi)$ defined above is an equational theory, and every nontrivial equational theory of commutative semigroups is of this form.

This result requires a few comments.

First, we note that in the presentation $E(J, m, r, \pi)=E(J, m, r) \cup \pi$ the union is disjoint: for $(\mathbf{a}, \mathbf{b}) \in E(J, m, r, \pi)$ either $\{\mathbf{a}, \mathbf{b}\} \subseteq J$ and $(\mathbf{a}, \mathbf{b}) \in E(J, m, r)$, or else $\{\mathbf{a}, \mathbf{b}\} \subseteq \Gamma^{+} \backslash J$ and $(\mathbf{a}, \mathbf{b}) \in \pi$. In particular, $E(J, m, r)$ can be characterized as the greatest $S$-theory contained in $E(J, m, r, \pi)$. A theory $E=E(J, m, r, \pi)$ is an $S$-theory itself, if the remainder $\pi$ has only trivial equations, i.e., is the least equivalence relation on $\Gamma_{+} \backslash J$.

It is the main idea of 11 that the difference $\pi=E \backslash E(J, m, r)$ is a relatively small part of $E$ with very special properties. While, in general, remainders may be very different (causing $\mathcal{L}(\mathrm{Com})$ itself to be quite complex), they have a "finite character", and can be described algorithmically.

We note that, in view of condition $(\pi 4)$, if $(\mathbf{a}, \mathbf{b}) \in \pi$, then the sequences $\mathbf{a}, \mathbf{b}$ are either equivalent or noncomparable in $\left(\Gamma_{+}, \leq\right)$. In particular, if $\mathbf{a}$ and $\mathbf{b}$ are not equal, then they have length $n \geq 2$. Moreover, by definition, the conditions $\left(C^{r}\right),\left(C_{m}\right)$ hold for each $(\mathbf{a}, \mathbf{b}) \in E(J, m, r, \pi)$. Fulfilling $\left(C_{m}\right)$ is related to the fact that $J \subseteq[(m)]$ is assumed in the definition of $E(J, m, r, \pi)$.

Finally, it should also be remarked that in [11], there are actually two sets of conditions denoted $(\mathrm{N} 1-\mathrm{N} 4)$ and $(\pi 1-\pi 4)$. These sets play independent roles in the proofs. However, in the final presentation, (N1, N2) can be omitted, since, as it was observed by M. Grech in [1], (N1, N2) follow from $(\pi 3, \pi 4)$. That is why we have decided to replace here $(\mathrm{N} 1-\mathrm{N} 4)$ by $\left(C^{r}\right)$ and $\left(C_{m}\right)$.

We will need the following description of algebraic operations in $\mathcal{L}(\mathrm{Com})$. Given a set $J$ and an equivalence relation $\pi$, by $C(J, \pi)$ we denote the union of all those blocks in $\pi$ which have a point in common with $J$, and by $\bar{C}(J, \pi)$ we denote the closure of $C(J, \pi)$ in $\Gamma$ under $\leq$-equivalence. (In fact, since by $(\pi 2), C(J, \pi)$ is closed under permutations, the only sequences we need to add are those obtained from sequences in $C(J, \pi)$ by adjoining zeros.) With $E^{S}$ denoting the greatest $S$ theory contained in $E$, and with superscripts denoting Cartesian powers, we have the following. 
Theorem 2.2. If $E_{1}=E\left(J_{1}, m_{1}, r_{1}, \pi_{1}\right)$ and $E_{2}=E\left(J_{2}, m_{2}, r_{2}, \pi_{2}\right) \in \mathcal{L}(\mathrm{Com})$, then the following hold.

(i) $E_{1} \supseteq E_{2}$ if and only if $J_{1} \supseteq J_{2}, m_{1} \leq m_{2}, r_{1}$ divides $r_{2}$, and $\pi_{1} \cup J_{1}^{2} \supseteq \pi_{2}$.

(ii) $E_{1} \vee E_{2}=E\left(J_{1} \cup J_{2} \cup \bar{C}, \min \left(m_{1}, m_{2}\right), \operatorname{gcd}\left(r_{1}, r_{2}\right),\left(\pi_{1} \vee \pi_{2}\right) \backslash C^{2}\right)$, where $C=C\left(J_{1} \cup J_{2}, \pi_{1} \vee \pi_{2}\right)$.

(iii) $E_{1} \cap E_{2}=E\left(J_{1} \cap J_{2}, \max \left(m_{1}, m_{2}\right), \operatorname{lcm}\left(r_{1}, r_{2}\right), \pi\right)$, where $\pi=\left(\pi_{1} \cap \pi_{2}\right) \cup\left(\pi_{1} \cap E_{2}^{S}\right) \cup\left(\pi_{2} \cap E_{1}^{S}\right)$.

We note that (ii) states, in particular, that $J_{1} \cup J_{2} \cup \bar{C}$ is a filter. The meet above is often denoted by $\wedge$ and the inclusion by $\leq$. Since this is the case in [6] and [10, in this paper we use these two notations interchangeably.

Other facts from [11, which we need to use only once in this paper, are recalled in an appropriate place (usually they follow easily from the general results stated above). Since using the above results usually leads to quite technical considerations, for the sake of clarity, more basic things, like reasoning concerning definability and order relations, will be treated in this paper rather informally.

\section{Definable Sets}

The fact that Schwabauer theories $E(J, m, r)$ form a maximal modular sublattice of $\mathcal{L}(\mathrm{Com})$ has been proved by E. Nelson 15. We call it the skeleton of $\mathcal{L}(\mathrm{Com})$, because in view of Theorem 2.2 it has a relatively simple structure reflecting the structure of the whole lattice. (Nelson used the term "the skeleton" for the sublattice of the theories $E([(k)], m, r)$.) We are going to show that both the sublattice of Schwabauer theories as a whole and each of these theories individually are definable in $\mathcal{L}(\mathrm{Com})$. This means, in particular, that the skeleton is fixed by all the automorphisms of $\mathcal{L}(\mathrm{Com})$.

First, we establish the simplest facts concerning definability of some sets and individual theories, recalling by the way some basic facts about the structure of $\mathcal{L}(\mathrm{Com})$. We follow the notation introduced by Nelson [15], and applied in [11, where the theories $E([(k)], m, r)$ were denoted simply $E(k, m, r)$.

The greatest element in $\mathcal{L}(\mathrm{Com})$ is the theory $E(1,0,1)=E(\Gamma, 0,1)$ consisting of all the equations. There are the following co-atoms: $S=E(1,1,1)$, the theory of semilattices; $A_{p}=E(1,0, p)$, the theory of Abelian groups of prime exponent $p$, for all $p \geq 2$; and the theory $N=E([(1,1)], 0,1)$ of nil-semigroups, defined by $x y=u z$. (This is generally well-known, and may be easily deduced from what is recalled in Section 2) Obviously, the set of co-atoms is definable.

We still may distinguish (in the first-order language) the set of those theories below the co-atoms, that are contained precisely in one co-atom.

First, note that if a theory $E=E(J, m, r, \pi)$ has $J \neq[(1)]$, then $J \subseteq[(1,1)]$, and therefore, by Theorem 2.2 (i), $E$ is contained in $N$, and also in one of $A_{p}$ or $S$, unless $m=0$ and $r=1$. In the latter case, $E$ is of the form $E=E(J, 0,1, \pi)$, and $N$ is the unique co-atom containing $E$.

If $J=[(1)]$, then $m \leq 1$, since by the definition of $E(J, m, r, \pi)$ we have $J \subseteq$ $[(m)]$. Also, by definition of the remainder, $\pi$ is trivial in this case, i.e., $E$ is of the form $E(J, m, r)$. Now, if $m=1$, then $r>1$, and $E$ is contained in $S$ and one of the $A_{p}$. If $m=0$, then $E$ is contained in two different co-atoms $A_{p}$ and $A_{q}$, unless $r=p^{k}$ is a power of a prime $p$, in which case $A_{p}$ is the unique co-atom containing E. 


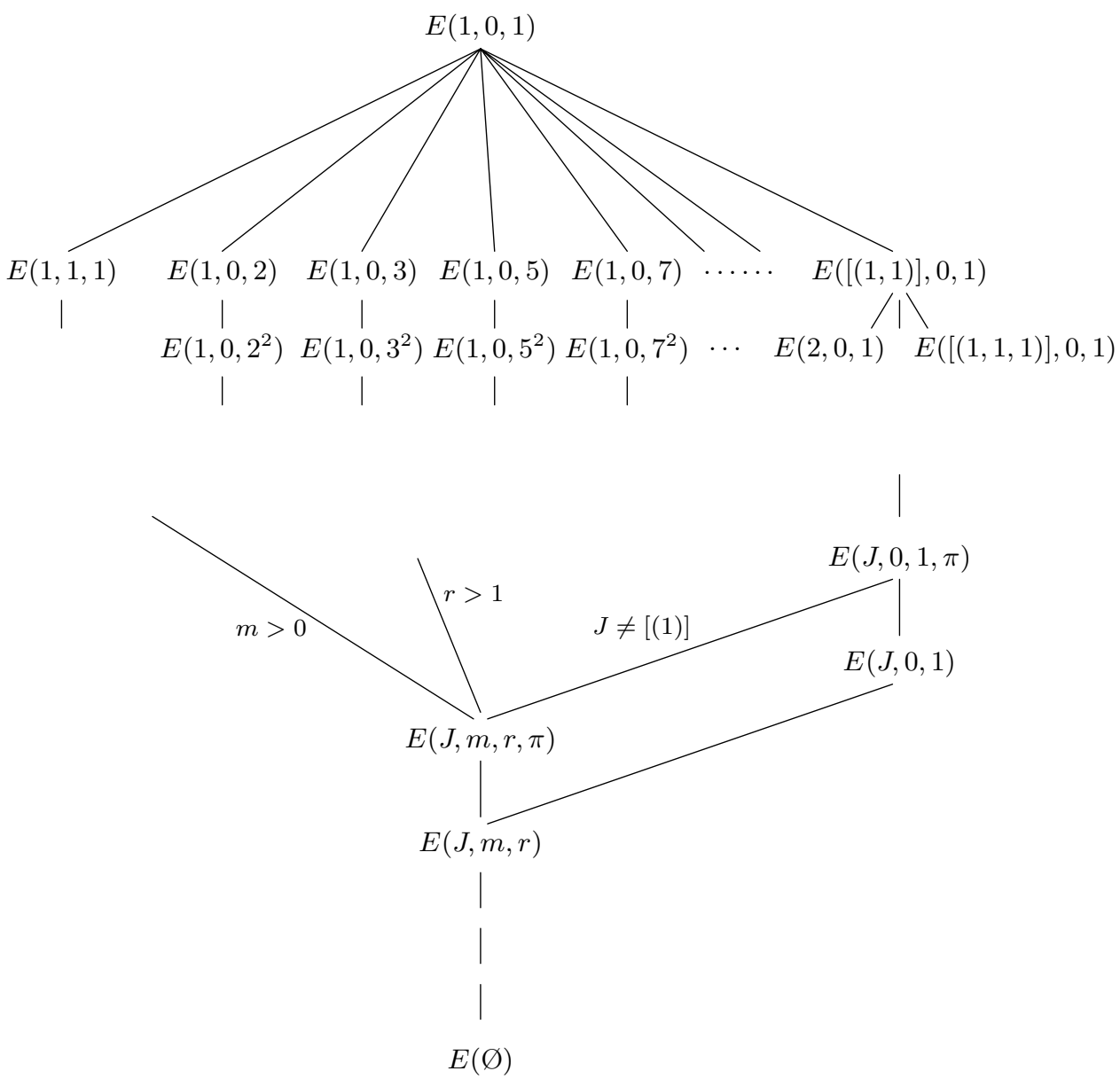

Figure 2. Co-atoms in $\mathcal{L}(\mathrm{Com})$ and the method of comparing filters.

Hence, the set $\mathcal{U}$ of the theories that are contained precisely in one co-atom consists of the theories of the form $E(J, 0,1, \pi)$ contained in $N$, and for each prime $p$, the theories $A_{p^{k}}=E\left(1,0, p^{k}\right)$ contained in $A_{p}$. At the present stage we are not able to distinguish (in the first-order language) between different $A_{p}$, but we can distinguish the theory $N$ from all $A_{p}$. Indeed, while there are many noncomparable theories $E(J, 0,1, \pi)$ in $\mathcal{U}$ contained in $N$, any two theories $E\left(1,0, p^{k}\right)$ contained in $A_{p}$, for a fixed $p$, are comparable. In other words, $\mathcal{U}$ consists of chains of theories $E\left(1,0, p^{k}\right)$ and a set of theories $E(J, 0,1), J \subseteq[(1,1)]$, order-isomorphic to the lattice of filters $J$ in $\Gamma$ (without the top and bottom elements), together with the companion theories $E(J, 0,1, \pi)$ with nontrivial remainder. The latter contains many noncomparable elements, for example, $E([(1,1,1)], 0,1)$ and $E(2,0,1)$ (see Figure 2, cf. Figure 1).

It follows that the theory $N$ is element-definable, and the set of theories of the form $E(J, 0,1, \pi)$ is definable. Also $S$ is element-definable (no element of $\mathcal{U}$ is contained in $S$ ), and therefore the set of all the theories contained in $S$ is definable. 
The latter coincides with the theories $E(J, m, r, \pi)$ with $m>0$, which is simply the set of regular theories (generated by regular identities). This means that the set of theories with $m=0$ is definable, as well. In turn, the theories with $r=1$ are those not contained in any co-atom $A_{p}$ (i.e. in any co-atom different from $N$ and $S)$. Hence, the set of these theories is definable. Consequently, the set of theories of the form $E(J, 0, r, \pi)$ with $r>1$ is definable. This set may be partitioned into the theories contained in $N$ (those with $J \neq[(1)]$ ) and the remaining, which are those of the form $E(1,0, r)$ ( $\pi$ is always trivial when $J=[(1)])$. The latter are precisely the theories $A_{r}=E(1,0, r)$ of Abelian groups.

We summarize these observations in the following proposition.

Proposition 3.1. The following individual theories and sets of theories are definable in $\mathcal{L}(\mathrm{Com})$ :

(a) the theory $N=E([(1,1)], 0,1)$ of nil-semigroups, and the set of theories of the form $E(J, 0,1, \pi)$;

(b) the theory $S=E(1,1,1)$ of semilattices, and the set of regular theories;

(c) the set of theories $A_{p}=E(1,0, p)$ of Abelian groups of prime exponent, and the set of all theories $A_{r}=E(1,0, r)$ of Abelian groups;

(d) the set of theories with $m=0$, and the set of theories with $r=1$.

Now we proceed to demonstrate that the set of all Schwabauer theories is definable. We make use of the set of all theories of the form $E(J, 0,1, \pi)$, which is definable by the above proposition. Let us denote this set by $\mathcal{N}$.

First we note that directly from the definition it follows that a remainder $\pi$ of type $(J, m, r)$, for any $m \geq 0$ and $r>0$, is also a remainder of type $(J, 0,1)$. Therefore, to every theory $E=E(J, m, r, \pi)$, we may assign uniquely a theory in $\mathcal{N}$, putting $M(E)=E(J, 0,1, \pi)$. Observe that, in view of Theorem 2.2 (i), $M(E)$ is actually the smallest theory in $\mathcal{N}$ containing $E$. Hence, this assignment can be expressed in the first-order language of $\mathcal{L}(\mathrm{Com})$.

Given a theory $E_{0}=E\left(J_{0}, 0,1, \pi_{0}\right)$ in $\mathcal{N}$, consider now the set $M^{-1}\left(E_{0}\right)$ of those theories $E \in \mathcal{L}(\mathrm{Com})$ for which $M(E)=E_{0}$. It consists of all theories of the form $E_{0}=E\left(J_{0}, m, r, \pi_{0}\right)$ such that $J_{0} \subseteq[(m)]$ and $\pi_{0}$ is a remainder of type $\left(J_{0}, m, r\right)$. If $\pi_{0}$ is trivial (contains no nontrivial equation), then the latter is no restriction, and in particular, $M^{-1}\left(E_{0}\right)$ has no minimal element. Namely, if $E\left(J_{0}, m, r\right) \in M^{-1}\left(E_{0}\right)$, then $E\left(J_{0}, m, 2 r\right) \in M^{-1}\left(E_{0}\right)$, as well. Otherwise, there are maximal $r$ and $m$ such that each $(\mathbf{a}, \mathbf{b}) \in \pi$ satisfies conditions $\left(C^{r}\right),\left(C_{m}\right)$, and therefore $M^{-1}\left(E_{0}\right)$ has the least element $E\left(J_{0}, m, r, \pi_{0}\right)$ with maximal $m$ and $r$.

It follows that for a theory $E=E(J, m, r, \pi)$, the remainder $\pi$ is trivial if and only if for every theory $T$ such that $M(T)=M(E)$ there exists a theory $S$ properly contained in $T$ with $M(S)=M(E)$. Thus we have

Theorem 3.2. The set of Schwabauer theories is definable in $\mathcal{L}(C o m)$.

Finally, we apply this theorem to show that comparing filters of the theories can be expressed in the language of $\mathcal{L}(\mathrm{Com})$.

Given a theory $E=E(J, m, r, \pi)$, define $J(E)=E(J, 0,1)$ (this is well-defined, since for every filter $J$ we have $J \subseteq[(0)]=\Gamma)$. Observing that $J(E)$ is the greatest Schwabauer theory contained in $M(E)$ (cf. Figure 2), we see that this assignment is definable, as well. Obviously, for any two theories $E_{1}, E_{2}$ with filters $J_{1}, J_{2}$, respectively, $J\left(E_{1}\right) \subseteq J\left(E_{2}\right)$ if and only if $J_{1} \subseteq J_{2}$. Hence, we obtain the following. 
Corollary 3.3. There exists a formula $\Psi\left(E_{1}, E_{2}\right)$ in the first-order language of $\mathcal{L}(\mathrm{Com})$ such that $\Psi\left(E_{1}, E_{2}\right)$ holds for two theories $E_{1}=E\left(J_{1}, m_{1}, r_{1}, \pi_{1}\right)$ and $E_{2}=E\left(J_{2}, m_{2}, r_{2}, \pi_{2}\right)$ if and only if $J_{1} \subseteq J_{2}$.

Thus, the property that the filter of a theory $E_{1}$ is contained in the filter of a theory $E_{2}$ is definable. Proving the analogous statement for parameters $m$ and $r$ requires more involved arguments, and we do it later.

\section{Abelian groups and Schwabauer theories}

The aim of this section is to prove that each individual Schwabauer theory is definable. We start from the Abelian groups. Recall that in Proposition 3.1 we have established that the set of these theories is definable.

Proposition 4.1. Each theory $A_{r}=E(1,0, r)$ of Abelian groups is definable in $\mathcal{L}(\mathrm{Com})$.

Proof. Let us denote by $I(J, m, r)$ the set of all theories of the form $E(J, m, r, \pi)$, for $J, m$, and $r$ fixed. Recall that this can be characterized as the set of all those theories that contain $E(J, m, r)$ as the greatest $S$-theory. (It is known that this is in fact an interval in $\mathcal{L}(\mathrm{Com})$, but we do not need this fact.)

We are going to show that there exists a definable correspondence between theories $A_{r}=E(1,0, r)$ and certain $S$-theories, such that the linear ordering of integers $r$ corresponds to the inclusion relation for theories. To this end, we will make use of the natural correspondence between $S$-theories and the intervals $I(J, m, r)$ defined above. Using it, we assign a unique filter $J_{r}$ to every integer $r \geq 2$.

Given $r \geq 2$, let $J$ be a maximal filter such that the set $I(J, 0, r)$ is nontrivial, i.e., has more than one element. This is equivalent to the fact that there exists a nontrivial remainder $\pi$ of type $(J, 0, r)$. For such a remainder, and a nontrivial $(\mathbf{a}, \mathbf{b}) \in \pi$, there exists an index $i$ such that $\alpha_{i} \neq \beta_{i}$, say, $\alpha_{i}<\beta_{i}$. Then, by $\left(C^{r}\right)$, the difference $\beta_{i}-\alpha_{i} \geq r$. Since $\mathbf{a}$ and $\mathbf{b}$ are noncomparable, their length is at least 2 , and therefore $\mathbf{b} \geq(1, r+1)$. It follows that $(1, r+1) \notin J$.

So, let $J_{r}$ be the greatest filter not containing $(1, r+1)$, i.e., the union of all filters with this property. It is not difficult to see that $J_{r} \subseteq J_{s}$ if and only if $r \geq s$. Moreover, the equivalence relation $\pi_{r}$ on $\Gamma_{+} \backslash J_{r}$, whose only nontrivial block is $\{(1, r+1),(r+1,1)\}$, is a remainder of type $\left(J_{r}, 0, r\right)$. It follows that $J_{r}$ is actually the greatest filter $J$ such that $I(J, 0, r)$ is nontrivial, or more generally, the greatest filter $J$ such that $I(J, m, r)$ is nontrivial for some $m \geq 0$.

Consequently, for a fixed $r, E_{r}=E\left(J_{r}, 0, r\right)$ is the greatest $S$-theory $E(J, m, r)$ such that the set $I(J, m, r)$ corresponding to it is nontrivial. Since each $S$-theory $E(J, m, r)$ is contained in $A_{r}=E(1,0, r)$, we obtain that $E_{r}=E\left(J_{r}, 0, r\right)$ may be defined as the greatest $S$-theory contained in $A_{r}=E(1,0, r)$ such that the set $I(J, m, r)$ corresponding to it is nontrivial (i.e., there exists a theory $E$, other than $E_{r}$ itself, having $E_{r}$ as the greatest $S$-theory contained in it). This can be expressed in the first-order language of $\mathcal{L}(\mathrm{Com})$.

Therefore, also $E_{r}^{*}=E\left(J_{r}, 0,1\right)$, the greatest $S$-theory having the same filter as $E_{r}$, may be assigned to $A_{r}$ in the first-order language, in view of Corollary 3.3

From the properties of the filters, $s \geq r$ if and only if $E_{s}^{*} \subseteq E_{r}^{*}$, for $r \geq 2$. Hence, the property that a theory of Abelian groups $A_{r}$ has smaller exponent than a theory of Abelian groups $A_{s}$ is definable in $\mathcal{L}(\mathrm{Com})$. Now, $A_{2}=E(1,0,2)$ is the theory of Abelian groups with the smallest exponent, and therefore, in view of 
Proposition 3.1 and the remark above, is definable. An easy induction yields the result.

We are now ready to deal with integer parameters $m$ and $r$. For a theory $E=$ $E(J, m, r, \pi)$ we put $m(E)=m$ and $r(E)=r$.

Proposition 4.2. Let $m_{0} \geq 0$ and $r_{0}>0$ be fixed integers. Then the following relations are definable in $\mathcal{L}(\mathrm{Com})$ :

(a) $r(E)=r_{0}$;

(b) $r\left(E_{1}\right)$ divides $r\left(E_{2}\right)$;

(c) $m(E)=m_{0}$;

(d) $m\left(E_{1}\right) \leq m\left(E_{2}\right)$;

(e) $r\left(E_{1}\right) \leq r\left(E_{2}\right)$.

Proof. First note that, in view of Theorem 3.2, we may restrict ourselves to $S$ theories, since the parameters $m$ and $r$ are the same for a theory and the greatest $S$-theory contained in it.

Obviously, for a fixed $r \geq 2, r(E)=r$ for a theory $E=E\left(J^{\prime}, m^{\prime}, r^{\prime}\right)$ if and only if $E$ is included in $A_{r}=E(1,0, r)$ and it is not included in any $A_{s}$ for $s>r$. Moreover, $r(E)=1$ if and only if $E$ is not included in any $A_{r}$. This, in view of Proposition 4.1 and the final part of its proof, establishes (a).

Obviously $r\left(E_{1}\right)$ divides $r\left(E_{2}\right)$ if and only if, for all theories $A_{r}$, the fact that $E_{1}$ is contained in $A_{r}$ implies that $E_{2}$ is contained in $A_{r}$. This establishes (b).

For c), we apply induction. First, $m(E)=0$ is definable by Proposition 3.1d. Suppose that $m(E)=n$ is definable for a fixed $n$. Then, for an $S$-theory $E$, $m(E)=n+1$ if and only if there exists an $S$-theory $E_{n}$ with the same parameters $J$ and $r$ as in $E$, and with $m\left(E_{n}\right)=n$, such that $E$ is the greatest $S$-theory properly contained in $E_{n}$, and having the same $J$ and $r$ parameters as $E_{n}$. Using Theorem 2.2 it is not difficult to check that this definition is as required. In view of Corollary [3.3 and (b) above, this can be expressed in the first-order language, proving (c).

Finally, since we do not use either (d) or (e) in this paper, the proofs of these facts are left to the reader as an exercise.

The main result of this section is

Theorem 4.3. Each $S$-theory $E(J, m, r)$ is definable in $\mathcal{L}(C o m)$.

Proof. In view of Proposition 4.2, we need only find a way to define the filter $J$. By Corollary 3.3, it is definable that two theories have the same filter; therefore it is enough to consider a sublattice of $\mathcal{L}(\mathrm{Com})$ consisting of the theories $E(J, 0,1)$. By Theorem 2.2, this is isomorphic to the lattice $\mathcal{F}$ of all filters on $(\Gamma, \leq$ ) (up to the least element).

In this lattice, the set $\mathcal{F}_{1}$ of join-irreducible elements is just the set of onegenerated filters (i.e., those generated by a single sequence). Since every filter is finitely generated, i.e., is a join of a finite number of one-generated filters, all we need to prove is that every one-generated filter is definable in the ordered set $\mathcal{F}_{1}$.

The latter is dually isomorphic to the ordered set $\Gamma^{*}$ of sequences in $(\Gamma, \leq)$, taken up to equivalence. To fix notation, we assume that $\Gamma^{*}$ consists of nonincreasing sequences of positive integers (see Figure 1), and our aim is to prove that every sequence $\mathbf{a} \in \Gamma^{*}$ is definable in $\Gamma^{*}$. Since this set is well-founded (has no infinite descending sequences), we may use induction. 
The sequence (1) is the least element in $\Gamma^{*}$.

We claim that each sequence $\mathbf{a}=\left(\alpha_{1}, \ldots, \alpha_{n}\right) \in \Gamma^{*}$, other than (1), is determined (up to permutation) by its dual covers, i.e., any two different nonincreasing sequences have different collections of dual covers (note that $\Gamma^{*}$ is not a lattice, so we cannot speak about joins and meets).

First, if $\alpha_{n}=1$ then $\mathbf{a}$ has two types of dual covers: one is $\mathbf{b}=\left(\alpha_{1}, \ldots, \alpha_{n-1}\right)$ with the property $h_{1}(\mathbf{b})=\mathbf{a}$, and others $\mathbf{c}$ have the property $g_{i j}(\mathbf{c})=\mathbf{a}$, up to permutation. While the sum of elements in each $\mathbf{c}$ is equal to $\sum \alpha_{i}$, the sum of elements in $\mathbf{b}$ is strictly less than that. Hence, in such a case, $\mathbf{a}$ is obtained from the dual cover with the lowest sum of elements, just by adjoining 1 to it.

Otherwise, all $\alpha_{i}>1$, and a can be obtained from the dual cover $\left(\gamma_{1}, \ldots, \gamma_{n+1}\right)$ for which the last entry $\gamma_{n+1}=1$ and $\gamma_{n}$ is the least possible. Indeed, in any case, this must be equal to the dual cover $\left(\alpha_{1}, \ldots, \alpha_{n-1}, \alpha_{n}-1,1\right)$. (Originally, this part of the proof was not so straight; the simplification is due to Ralph McKenzie.)

Thus, our claim is proved. It easily follows from it that each element of $\Gamma^{*}$ can be individually defined in the first-order language of $\Gamma^{*}$.

\section{Definability up to Permutation}

A natural way to prove that each finitely axiomatizable theory is definable, is to prove that each one-based theory (i.e., axiomatized, or as we rather say, generated by a single equation) is definable. This is, obviously, equivalent to proving that for each equation $(\mathbf{a}, \mathbf{b})$ the fact that $(\mathbf{a}, \mathbf{b}) \in E$ can be expressed in the first-order language of $\mathcal{L}(\mathrm{Com})$.

We have not been able to prove this, because, as we show in Section 7, this is not true in general. Instead, we prove some closely related facts, which help us to prove the definability of many individual theories with nontrivial remainders. Namely, we show that, given an equation $(\mathbf{a}, \mathbf{b})$, the properties that $(\mathbf{a}, p(\mathbf{b})) \in E$ for some permutation $p$, and that $E$ is generated by a set of equations of the form $(\mathbf{a}, p(\mathbf{b}))$, where $p$ is a permutation, are definable in $\mathcal{L}(\mathrm{Com})$. We have already mentioned in the introduction that, in the case of commutative semigroups, there is no natural way to distinguish permutations of sequences leading to different equations. The results of this section show that this is, in fact, the crucial problem.

Let us recall first from [11] that if $E\left(J_{0}, m_{0}, r_{0}, \pi_{0}\right)$ is a theory generated by a single equation $(\mathbf{a}, \mathbf{b})$, then $m_{0}$ is the largest $m$ such that $(\mathbf{a}, \mathbf{b})$ satisfies condition $\left(C_{m}\right)$, and $r_{0}$ is the largest $r$ such that condition $\left(C^{r}\right)$ is satisfied. Moreover, if sequences $\mathbf{a}, \mathbf{b}$ are comparable and nonequivalent, say $\mathbf{a}<\mathbf{b}$, then the equation $(\mathbf{a}, \mathbf{b})$ generates an $S$-theory $E(J, m, r)$ with $J=[\mathbf{a}]$ (in fact, these observations may be inferred from the general results recalled in Section 2. It follows that the theories generated by a single equation $(\mathbf{a}, \mathbf{b})$ with comparable and nonequivalent sequences are individually definable in $\mathcal{L}(\mathrm{Com})$.

Further, recall that an equation $(\mathbf{a}, \mathbf{b})$ is regular if $\alpha_{i}, \beta_{i}>0$ for all $i$. For nonregular equations $(\mathbf{a}, \mathbf{b})$, it is easy to observe (cf. [11, $\S 3.16]$ ) that each such equation generates an $S$-theory $E(J, m, r)$ with $J=[\mathbf{a}, \mathbf{b}]$ and $m=0$. Hence, each theory generated by a nonregular equation is definable, as well.

So, we may assume that equations we deal in the remainder of this section are regular, and we distinguish two cases according to whether sequences in the equation are equivalent or not. Nontrivial regular equations with equivalent sequences (i.e., 


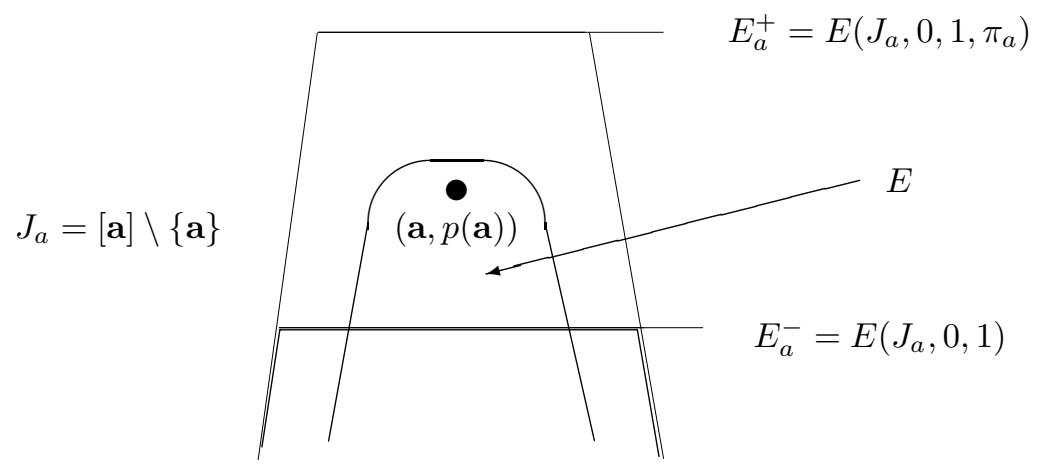

Figure 3. Proof of Theorem 5.1.

equations of the form $(\mathbf{a}, p(\mathbf{a}))$, where $p \in S_{n}$ is a nontrivial permutation and $\mathbf{a}$ is a sequence of positive integers) are called permutational.

For a fixed sequence a of positive integers, we define some filters, remainders, and theories. Let $J_{a}=[\mathbf{a}] \backslash\{\mathbf{a}\}$; this is the largest filter contained in [a] and not containing a. Let $E_{a}^{-}=E\left(J_{a}, 0,1\right)$, and let $\pi_{a}$ be the remainder of type $\left(J_{a}, 0,1\right)$ whose only nontrivial block is one consisting of all the sequences $p(\mathbf{a})$, where $p$ is a permutation (the conditions $(\pi 1-\pi 4)$ are obviously satisfied). Let $E_{a}^{+}=E\left(J_{a}, 0,1, \pi_{a}\right)$. It can be characterized as the largest theory contained in $E([\mathbf{a}], 0,1)$ that has the same filter as $E_{a}^{-}$. Since all $S$-theories are definable, by Corollary [3.3, $E_{a}^{+}$is definable. We make use of these facts in the proof below.

Theorem 5.1. Given a sequence a of positive integers, the following properties are definable in $\mathcal{L}(\mathrm{Com})$ :

(i) a nontrivial equation $(\mathbf{a}, p(\mathbf{a})) \in E$ for some permutation $p$; and

(ii) $E$ is generated by a set of permutational equations of the form $(\mathbf{a}, p(\mathbf{a}))$.

Proof. For (i), note that $(\mathbf{a}, p(\mathbf{a})) \in E$ for some $p$ if and only if $E \cap E_{a}^{+} \not \subset E_{a}^{-}$(see Figure (3). Since $E_{a}^{+}$and $E_{a}^{-}$are definable, this proves the claim.

For (ii), let $\Phi(E)$ abbreviate the following formula in the language of $\mathcal{L}(\mathrm{Com})$ :

$$
E \subseteq E_{a}^{+} \text {, and if } E=E_{1} \vee E_{2} \text { and } E_{2} \subseteq E_{a}^{-} \text {, then } E_{1}=E \text {. }
$$

Using Theorem 2.2 repeatedly we show that $\Phi(E)$ holds if and only if $E$ satisfies (ii).

First, assume that $E=E(J, m, r, \pi)$ is a theory generated by a set of equations of the form $(\mathbf{a}, p(\mathbf{a}))$. Then, obviously, $E \subseteq E_{a}^{+}$, in particular $J \subseteq J_{a}$, and moreover, $E$ is generated by equations in $\pi \cap \pi_{a}$. Suppose that $E=E_{1} \vee E_{2}$ with $E_{2} \subseteq E_{a}^{-}$. Then $E_{1} \subseteq E$, and consequently, $J_{1} \subseteq J \subseteq J_{a}$. Next, $E \subseteq E_{1} \vee E_{a}^{-}=E\left(J_{a}, 0,1, \pi_{1} \backslash J_{a}^{2}\right)$, where $\pi_{1}$ is the remainder of $E_{1}$. It follows that $\pi \subseteq\left(\pi_{1} \backslash J_{a}^{2}\right) \cup J_{a}^{2}$, and consequently, $\pi \cap \pi_{a} \subseteq \pi_{1}$ (since there is no nontrivial identity in $\pi_{a} \cap J_{a}^{2}$ ). The latter implies that the generating set for $E$ is contained in $E_{1}$, which, in view of $E \supseteq E_{1}$, proves that $E=E_{1}$, and in consequence, that $\Phi(E)$ holds.

Conversely, suppose that $\Phi(E)$ is satisfied for $E=E(J, m, r, \pi)$. Then, taking $E_{2}=E \cap E_{a}^{-}$, and $E_{1}$ the theory generated by $E \backslash E_{a}^{-}$, we get $E=E_{1} \vee E_{2}$. By the second condition in $\Phi(E), E=E_{1}$. Since by the first condition $E \backslash E_{a}^{-}$contains only equations of the form $(q(\mathbf{a}), p(\mathbf{a}))$, condition (ii) follows. 
Example 5.2. Let $\mathbf{a}=(1,2)$. Then $J_{a}=[(1,1,2),(3)]$, and $\pi_{a}$ has one nontrivial block $\{(1,2),(2,1)\}$. Consequently, the only theory generated by a set of permutational equations of the form $(\mathbf{a}, p(\mathbf{a}))$ is one generated by $((1,2),(2,1))$. Hence, this theory is definable. Note that it is different from $E_{a}^{+}$, since the equation $((3),(4))$ in one variable is not a consequence of $((1,2),(2,1))$.

Example 5.3. Let $\mathbf{a}=(1,2,3)$. Then $J_{a}=[(1,1,2,3),(3,3),(2,4),(1,5)]$, and the nontrivial block of $\pi_{a}$ consists of 6 permutations of the sequence $(1,2,3)$. There are six theories generated by a set of equations of the form $(\mathbf{a}, p(\mathbf{a}))$ in this case. These include: $E_{1}, E_{2}, E_{3}$, and $E_{4}$ generated by single equations $((1,2,3),(2,3,1))$, $((1,2,3),(1,3,2)),((1,2,3),(3,2,1))$, and $((1,2,3),(2,1,3))$, respectively, $E_{5}$ generated by all equations $(\mathbf{a}, p(\mathbf{a}))$, and the trivial theory $E_{0}$. They correspond to the subgroups of the symmetric group $S_{3}$.

It is not difficult to see that all these theories are individually definable. Indeed, the trivial theory is definable, and $E_{5}$ is definable, in view of Theorem5.1(ii), as the greatest theory generated by a set of equations of the form (a, $p(\mathbf{a}))$. Consequently, the set of the remaining theories $E_{1}, E_{2}, E_{3}, E_{4}$ is definable.

Now, among these theories, $m\left(E_{2}\right)=2$, while for the other theories $m\left(E_{i}\right)=1$. It follows, in view of Proposition 4.2 that $E_{2}$ is definable. Similarly, $r\left(E_{3}\right)=$ 2, while for the other theories $r\left(E_{i}\right)=1$. Hence, also $E_{3}$ is definable. Finally, applying $g_{13}$, we get that $((4,2),(3,3)) \in E_{1}$, while it may be checked directly (or using the method given in [11]) that $((4,2),(3,3)) \notin E_{4}$. Consequently, the theory $E^{*}$ generated by $((4,2),(3,3))$ is contained in $E_{1}$, and not contained in $E_{4}$. In Example 5.5 below, we will see that $E^{*}$ is definable. This implies that also $E_{1}$ and $E_{4}$ are definable.

As the second case, we consider regular equations $(\mathbf{a}, \mathbf{b})$ with noncomparable sequences $\mathbf{a}$ and $\mathbf{b}$. So, for the remainder of this section, we assume that $\mathbf{a}, \mathbf{b}$ are fixed, noncomparable sequences of positive integers, of the same length.

As before, we start from some analogous definitions. Let $J_{a b}=[\mathbf{a}, \mathbf{b}] \backslash\{\mathbf{a}, \mathbf{b}\}$, which is the largest filter contained in $[\mathbf{a}, \mathbf{b}]$, not containing $\mathbf{a}, \mathbf{b} ; E_{a b}^{-}=E\left(J_{a b}, 0,1\right)$, and let $\pi_{a b}$ be the remainder of type $\left(J_{a b}, 0,1\right)$ whose only nontrivial block is one consisting of all the sequences $p(\mathbf{a})$ and $q(\mathbf{b})$ for permutations $p, q$. Again, it is easily seen that $\pi_{a b}$ is a well-defined remainder, and $E_{a b}^{+}=E\left(J_{a b}, 0,1, \pi_{a b}\right)$ is definable as the largest theory contained in $E([\mathbf{a}, \mathbf{b}], 0,1)$ that has the same filter as $E_{a b}^{-}$.

In this case we define one more theory $E_{a b}=E_{a}^{+} \vee E_{b}^{+} \vee E_{a b}^{-}$. Then, by the previous results on permutational equations, $E_{a b}$ is definable in $\mathcal{L}(\mathrm{Com})$, and since $\mathbf{a}, \mathbf{b}$ are noncomparable, $E_{a b}=E\left(J_{a b}, 0,1, \pi_{a b}^{*}\right)$, where $\pi_{a b}^{*}$ has exactly two nontrivial blocks consisting of all the sequences $p(\mathbf{a})$ in one block, and all the sequences $q(\mathbf{b})$, in the other.

Theorem 5.4. Given noncomparable sequences $\mathbf{a}, \mathbf{b}$ of positive integers, the following properties are definable in $\mathcal{L}(\mathrm{Com})$ :

(i) an equation $(\mathbf{a}, p(\mathbf{b})) \in E$ for some permutation $p$; and

(ii) $E$ is generated by a set of equations of the form $(\mathbf{a}, p(\mathbf{b}))$.

Proof. For (i), as before, it is enough to note that $(\mathbf{a}, p(\mathbf{b})) \in E$ for some $p$ if and only if $E \cap E_{a b}^{+} \not \subset E_{a b}$ (see Figure 4 ).

For (ii), now let $\Phi(E)$ abbreviate the following formula:

$$
E \subseteq E_{a b}^{+} \text {, and if } E=E_{1} \vee E_{2} \text { and } E_{2} \subseteq E_{a b}^{-} \text {, then } E_{1}=E \text {. }
$$




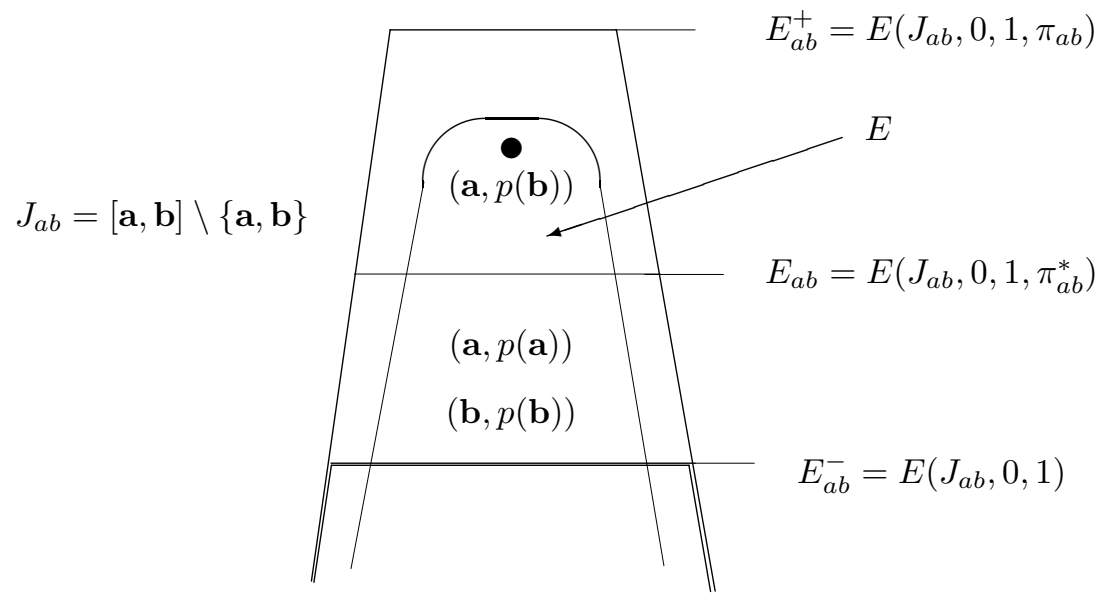

Figure 4. Proof of Theorem 5.4.

Let $P=P(\mathbf{a}, \mathbf{b})$ denote the set of all equations of the form $(\mathbf{a}, p(\mathbf{a})),(\mathbf{a}, p(\mathbf{b}))$, or $(\mathbf{b}, q(\mathbf{b}))$, where $p$ is a permutation. We first show that $\Phi(E)$ holds if and only if $E$ is generated by a set of permutational equations contained in $P$. The proof is essentially the same as that of Theorem 5.1 (ii).

First, assume that $E=E(J, m, r, \pi)$ is a theory generated by a set of equations contained in $P$. Then $E \subseteq E_{a b}^{+}$, in particular $J \subseteq J_{a b}$, and moreover, $E$ is generated by equations in $\pi \cap \pi_{a b}$. Suppose that $E=E_{1} \vee E_{2}$ with $E_{2} \subseteq E_{a b}^{-}$. Then $E \subseteq E_{1} \vee E_{a b}^{-}=E\left(J_{a b}, 0,1, \pi_{1} \backslash J_{a b}^{2}\right)$, where $\pi_{1}$ is the remainder of $E_{1}$. It follows that $\pi \subseteq\left(\pi_{1} \backslash J_{a b}^{2}\right) \cup J_{a b}^{2}$, and consequently, $\pi \cap \pi_{a b} \subseteq \pi_{1}$. The latter implies that $E \subseteq E_{1}$, and consequently $E=E_{1}$, as required.

Conversely, suppose that $\Phi(E)$ is satisfied for $E=E(J, m, r, \pi)$. Then, taking $E_{2}=E \cap E_{a b}^{-}$, and $E_{1}$ the theory generated by $E \backslash E_{a b}^{-}$, we get $E=E_{1} \vee E_{2}$, which implies $E=E_{1}$. Since by the first condition in $\Phi(E)$ we have $E \backslash E_{a b}^{-} \subseteq P$, the claim follows.

Now, to complete the proof, note that a pair of equations $(\mathbf{a}, p(\mathbf{b})),(\mathbf{b}, q(\mathbf{b}))$ is equivalent to $(\mathbf{a}, p(\mathbf{b})),(\mathbf{a}, p q(\mathbf{b}))$, while the pair $(\mathbf{a}, p(\mathbf{b})),(\mathbf{a}, q(\mathbf{a}))$ is equivalent to $(\mathbf{a}, p(\mathbf{b})),(\mathbf{a}, q p(\mathbf{b}))$. It follows that the theories satisfying (ii) are precisely the theories satisfying (i) and $\Phi(E)$.

Example 5.5. Let $\mathbf{a}=(4,2)$ and $\mathbf{b}=(3,3)$. Then $J_{a b}=[(1,2,4),(1,3,3),(6)]$; the nontrivial block of $\pi_{a b}$ is $\{(4,2),(2,4),(3,3)\}$; and $\pi_{a b}^{*}$ also has only one nontrivial block $\{(4,2),(2,4)\}$ (since the block with $(3,3)$ is trivial). In this case the theory $E^{*}$ generated by $(\mathbf{a}, \mathbf{b})$ is the only theory contained in $E_{a b}^{+}$generated by equations of the form $(\mathbf{a}, p(\mathbf{b}))$. Hence, this theory is definable. In Section[7, we show however that this is not generally the case for theories generated by a single equation in two variables.

Many one-based theories generated by a regular equation $(\mathbf{a}, \mathbf{b})$ with noncomparable sequences may be proved to be definable, following Example 5.3 Generally, this depends on particular properties of the set of the theories generated by a set 
of equations of the form $(\mathbf{a}, p(\mathbf{b}))$ or $(\mathbf{a}, p(\mathbf{a}))$. In the next section, we look for a general result in this direction.

\section{DefinABle THEORIES WITH NONTRIVIAL REMAINDERS}

From remarks at the beginning of the previous section it follows that each onebased theory generated by an irregular equation, or by an equation with comparable but nonequivalent sequences, is an $S$-theory (i.e., its remainder is trivial), and therefore, in view of Theorem 4.3, is definable in $\mathcal{L}(\mathrm{Com})$. In this section we consider one-based theories with nontrivial remainders.

According to Theorem [5.4, we are able to express the fact that a given equation $(\mathbf{c}, \mathbf{d})$ is in a theory $E$ up to permutation, i.e., $(\mathbf{c}, p(\mathbf{d})) \in E$ for some permutation $p$.

The idea applied below is to make use of consequences $(\mathbf{a}, \mathbf{b})$ of a fixed equation $(\mathbf{c}, \mathbf{d})$, as in Example 5.3 in hope that the set of all pairs $(\mathbf{a}, p(\mathbf{b}))$ associated with those consequences determines the theory generated by $(\mathbf{c}, \mathbf{d})$ uniquely. This turns out to be true for a large class of theories with a nontrivial remainder.

A regular equation $(\mathbf{a}, \mathbf{b})$ is called balanced, if the covers of $\mathbf{a}$ are noncomparable with the covers of $\mathbf{b}$. In particular, $\mathbf{a}, \mathbf{b}$ have to be noncomparable. (In fact, we may restrict to $g_{i j}$-covers in this definition. This can be proved using the alternate definition of the order $(\Gamma, \leq)$ given in [11]. Since we make no use of this fact in our proofs below, we leave the proof to the reader.)

It is often the case that an equation $(\mathbf{a}, \mathbf{b})$ with noncomparable sequences is balanced. For example, if $\sum \mathbf{a}=\sum \mathbf{b}$ (the sums of elements in the sequences are equal), then it is enough that up to permutation the sequences differ in more than 3 elements. If $\sum \mathbf{a}<\sum \mathbf{b}$, then it is enough that $\mathbf{a}$ has an element $\alpha_{i}$ greater than the sum $\beta_{r}+\beta_{s}$ of any two elements in $\mathbf{b}$. On the other hand, no equation in two variables is balanced (we consider equations in two variables in the next section).

In this section, let us assume that $(\mathbf{c}, \mathbf{d})$ is a fixed balanced equation in $n>2$ variables, and $E(\mathbf{c}, \mathbf{d})$ denotes the theory generated by $(\mathbf{c}, \mathbf{d})$. We shall consider only certain special consequences of $(\mathbf{c}, \mathbf{d})$ : the set $C_{0}$ of consequences in $n$ variables obtained from $(\mathbf{c}, \mathbf{d})$ by permutations and transitivity only, and the set $C_{1}$ of consequences in $n-1$ variables obtained from $(\mathbf{c}, \mathbf{d})$ by identifying exactly two variables in any $(\mathbf{a}, \mathbf{b}) \in C_{0}$, and applying permutations and transitivity. In particular, we do not apply multiplication (i.e., operation $h_{1}$ ). To prove properties of $C_{0}$ and $C_{1}$ we will need later, let us start from more formal definitions.

Let $C_{0}=C_{0}(\mathbf{c}, \mathbf{d})$ be the least set of pairs containing $(\mathbf{c}, \mathbf{d})$ that is an equivalence relation and closed under permutations (in the sense of condition $(\pi 2)$ ). Let $C_{1}=$ $C_{1}(\mathbf{c}, \mathbf{d})$ be the least set of pairs, containing all sets $g_{i j}\left(C_{0}\right)$, that is an equivalence relation and closed under permutations (similarly as in the case of permutations, operations $g_{i j}$ are applied to every pair in $C_{0}$, to both the sequences in the pair simultaneously, and $i, j$ are allowed to run over all possible indices). Since, the equation $(\mathbf{c}, \mathbf{d})$ is assumed to be fixed throughout this section, we will usually use the short notation $C_{0}$ and $C_{1}$.

Our first observation is

Lemma 6.1. The set $C_{0}$ is the set of all equations $(\mathbf{a}, \mathbf{b}) \in E(\mathbf{c}, \mathbf{d})$ in $n$ variables such that both $\mathbf{a}$ and $\mathbf{b}$ are equivalent to $\mathbf{c}$ or $\mathbf{d}$. The set $C_{1}$ is the set of all equations $(\mathbf{a}, \mathbf{b}) \in E(\mathbf{c}, \mathbf{d})$ in $n-1$ variables such that both $\mathbf{a}$ and $\mathbf{b}$ are $g_{i j}$-covers of $\mathbf{c}$ or $\mathbf{d}$. 
Proof. Obviously $C_{0}$ and $C_{1}$ are contained, respectively, in the sets of equations described in the lemma. To show the converse inclusions it is enough to construct a theory $E^{\prime}$ containing $(\mathbf{c}, \mathbf{d})$ such that if $(\mathbf{a}, \mathbf{b}) \in E$ and both $\mathbf{a}$ and $\mathbf{b}$ either are equivalent to $\mathbf{c}$ or $\mathbf{d}$, or are $g_{i j}$-covers of $\mathbf{c}$ or $\mathbf{d}$, then $(\mathbf{a}, \mathbf{b}) \in C_{0} \cup C_{1}$.

To this end, let $J^{\prime}$ be the filter generated by all the covers $g_{i j}(\mathbf{c}), h_{1}(\mathbf{c}), g_{i j}(\mathbf{d})$, $h_{1}(\mathbf{d})$, and let $J$ be the set obtained from $J^{\prime}$ by removing all the elements $g_{i j}(\mathbf{c})$, $g_{i j}(\mathbf{d})$. Since they are pairwise noncomparable (by assumption that $(\mathbf{c}, \mathbf{d})$ is balanced), $J$ is a filter, and moreover, both $h_{1}(\mathbf{c}), h_{1}(\mathbf{d}) \in J$.

Now, let $\pi$ be the union of $C_{0}$ and $C_{1}$, endowed with trivial pairs (a, a) for all $\mathbf{a} \in \Gamma_{+} \backslash J$. Then, it is easily seen that $\pi$ is a remainder of type $(J, 0,1)$. This follows from the facts that $J$ is the largest filter not containing $g_{i j}(\mathbf{c}), g_{i j}(\mathbf{d})$, while $h_{1}(\mathbf{c}), h_{1}(\mathbf{d}) \in J$, and that $C_{0}, C_{1}$ are equivalence relations closed under permutations. Clearly, the theory $E^{\prime}=E(J, 0,1, \pi)$ has the required properties.

We wish to describe $C_{0}$ in a direct way. To this end, for $\mathbf{c}=\left(\gamma_{1}, \ldots, \gamma_{n}\right)$ and $\mathbf{d}=$ $\left(\delta_{1}, \ldots, \delta_{n}\right)$, let $\Pi_{c}$ be the equivalence relation on the set of indices $I=\{1,2, \ldots, n\}$ such that $i, j$ are $\Pi_{c^{-}}$-equivalent if and only if $\gamma_{i}=\gamma_{j}$, and let $\Pi_{d}$ be the equivalence relation on the set $I$ such that $i, j$ are $\Pi_{d}$-equivalent if and only if $\delta_{i}=\delta_{j}$. Let $\Pi=\Pi(\mathbf{c}, \mathbf{d})$ be the least equivalence relation containing both $\Pi_{c}$ and $\Pi_{d}$.

We define $G(\mathbf{c}, \mathbf{d})$ to be the permutation group on the set of indices $I=\{1,2, \ldots$, $n$ \} generated by all transpositions $(i, j)$ with $i, j$ lying in the same block of $\Pi$. In other words, this is the group consisting of all permutations leaving all $\Pi$-blocks invariant, and it has the form of the direct product of the symmetric groups on blocks of $\Pi$.

Lemma 6.2. The set $C_{0}=C_{0}(\mathbf{c}, \mathbf{d})$ is the least symmetric and permutation closed relation containing all the pairs $(\mathbf{c}, p(\mathbf{d})),(\mathbf{c}, p(\mathbf{c}))$, and $(\mathbf{d}, p(\mathbf{d}))$ with $p \in G(\mathbf{c}, \mathbf{d})$.

Proof. Denote by $G(\mathbf{c})$ the group leaving all blocks of $\Pi_{c}$ invariant, and suppose first that a permutation $p \in G(\mathbf{c})$. Then $\left(\gamma_{p(1)} \ldots \gamma_{p(n)}\right)=\left(\gamma_{1}, \ldots, \gamma_{n}\right)$, and therefore the following are consequences of $(\mathbf{c}, \mathbf{d}):(\mathbf{c}, p(\mathbf{c})),(\mathbf{d}, p(\mathbf{c})),\left(p^{-1}(\mathbf{d}), \mathbf{c}\right),\left(p^{-1}(\mathbf{d}), \mathbf{d}\right)$, $(\mathbf{d}, p(\mathbf{d}))$. Analogous argument shows that the same holds for all $p \in G(\mathbf{d})$. It follows easily that $(\mathbf{c}, p(\mathbf{c})),(\mathbf{d}, p(\mathbf{d}))$, and $(\mathbf{c}, p(\mathbf{d}))$ are among the consequences of $(\mathbf{c}, \mathbf{d})$ for all $p \in G(\mathbf{c}, \mathbf{d})$.

The least symmetric and permutation closed relation $R$ containing all these pairs is the set of all pairs $\left(q p_{1}(x), q p_{2}(y)\right)$, where $p_{1}, p_{2} \in G(\mathbf{c}, \mathbf{d}), q$ is any permutation, and $x, y \in\{\mathbf{c}, \mathbf{d}\}$. Obviously, $R$ is reflexive. So, to see it equals $C_{0}$, it remains to observe that $R$ is also transitive.

This is easy. Suppose that $\left(q p_{1}(x), q p_{2}(y)\right)$ and $\left(f p_{3}(x), f p_{4}(y)\right)$ are in $R$, and $q p_{2}(x)=f p_{3}(y)$. Then, since $\mathbf{c}, \mathbf{d}$ are noncomparable, $x=y$, and $p_{2}^{-1} q^{-1} f p_{3}=$ $g \in G(\mathbf{c}, \mathbf{d})$ (since $g \in G(x)$ ). It follows that $q^{-1} f=p_{2} g p_{3}^{-1} \in G(\mathbf{c}, \mathbf{d})$. Hence, $\left(p_{1}(x), q^{-1} f p_{4}(y)\right) \in R$, and consequently, $\left(q p_{1}(x), f p_{4}(y)\right) \in R$, as required.

A direct description of $C_{1}$ is more complicated, but we shall need only a certain property of $C_{1}$. The following observation is crucial.

Lemma 6.3. Let $\mathbf{a}=\left(\alpha_{1}, \ldots, \alpha_{n}\right)$ be a sequence of positive integers. If $g_{i j}(\mathbf{a})$ and $g_{r s}(\mathbf{a})$ are equivalent (i.e., are the same up to permutation of elements), then $\left\{\alpha_{i}, \alpha_{j}\right\}=\left\{\alpha_{r}, \alpha_{s}\right\}$.

Proof. Consider the sequences a and $g_{i j}(\mathbf{a})$. If we treat these sequences as multisets (with possible repetitions of elements), then removing simultaneously equal 
elements from both the sets leaves us with $\left\{\alpha_{i}, \alpha_{j}\right\}$ in the first set, and $\left\{\alpha_{i}+\alpha_{j}\right\}$ in the second. This does not depend on the choice of a pair to be removed in a given step, since even if there is some $\alpha_{m}$ with $\alpha_{m}=\alpha_{i}+\alpha_{j}$, then the number of such $\alpha$ 's in the first set is always one less than in the second set.

In view of this lemma, from each $g_{i j}$-cover a of sequences $\mathbf{c}$ or $\mathbf{d}$, even if the cover is given up to permutation, we may infer the indices $\{i, j\}$, at least up to $\Pi_{c^{-}}$or $\Pi_{d^{-}}$-equivalence, respectively. Note that, in view of the assumption that $\mathbf{c}, \mathbf{d}$ are noncomparable, we are able to infer whether $\mathbf{a}$ is actually a $g_{i j}$-cover of $\mathbf{c}$ or a $g_{i j}$-cover of $\mathbf{d}$. It follows that, if $\mathbf{a}=p g_{i j}(\mathbf{c})$ or $\mathbf{a}=p g_{i j}(\mathbf{d})$, for a permutation $p$ on $n-1$ letters, we may infer which of these two cases occurs, and what the indices $i, j$ are, up to $\Pi$-equivalence. Hence, for any sequence $\mathbf{a}$, as above, we may define $P(\mathbf{a})$ uniquely to be the union $[i] \cup[j]$ of $\Pi$-equivalence classes representing $i$ and $j$, respectively. Note that this definition depends on the sequences $\mathbf{c}, \mathbf{d}$ (since $\Pi$ does). The property of $C_{1}$ we need is the following.

Lemma 6.4. If $(\mathbf{a}, \mathbf{b}) \in C_{1}$, then $P(\mathbf{a})=P(\mathbf{b})$.

Proof. By definition, $C_{1}$ is the least set of pairs containing all sets $g_{i j}\left(C_{0}\right)$ that is an equivalence relation and closed under permutations.

First suppose that $(\mathbf{a}, \mathbf{b}) \in g_{i j}\left(C_{0}\right)$ for some $i, j$, that is, $(\mathbf{a}, \mathbf{b})=\left(g_{i j}\left(\mathbf{a}_{1}\right), g_{i j}\left(\mathbf{a}_{2}\right)\right)$ for some $\left(\mathbf{a}_{1}, \mathbf{a}_{2}\right) \in C_{0}$. By Lemma 6.2. we may assume that $\left(\mathbf{a}_{1}, \mathbf{a}_{2}\right)=(f(\mathbf{c}), f p(\mathbf{d}))$ for some permutation $p \in G(\mathbf{c}, \mathbf{d})$, and a permutation $f$ on $n$ letters (other cases are analogous). Then, assuming $\mathbf{c}=\left(\gamma_{1}, \ldots, \gamma_{n}\right)$ and $\mathbf{d}=\left(\delta_{1}, \ldots, \delta_{n}\right)$, we compute:

$$
\mathbf{a}=g_{i j} f\left(\gamma_{1}, \ldots, \gamma_{n}\right)=g_{i j}\left(\gamma_{f(1)}, \ldots, \gamma_{f(n)}\right)=p_{1} g_{r s}\left(\gamma_{1}, \ldots, \gamma_{n}\right),
$$

where $r=f(i)$, and $s=f(j)$, and $p_{1}$ is a suitable permutation on $n-1$ letters obtained from $f$. Similarly,

$$
\begin{aligned}
\mathbf{b} & =g_{i j} f p\left(\delta_{1}, \ldots, \delta_{n}\right)=g_{i j} f\left(\delta_{p(1)}, \ldots, \delta_{p(n)}\right)=g_{i j}\left(\delta_{p f(1)}, \ldots, \delta_{p f(n)}\right) \\
& =p_{2} g_{u v}\left(\delta_{1}, \ldots, \delta_{n}\right),
\end{aligned}
$$

where $u=p f(i)$ and $v=p f(j)$, and $p_{2}$ is a suitable permutation on $n-1$ letters obtained from $p f$. (Note that, by definition, applying the product of permutations to a sequence corresponds to applying the permutations to the indices in the reverse order!)

Using the fact that $p \in G(\mathbf{c}, \mathbf{d})$, we obtain that $[f(i)]=[p f(i)]$ and $[f(j)]=$ $[p f(j)]$, and consequently, $P(\mathbf{a})=P(\mathbf{b})$. An analogous argument for other cases yields that if $(\mathbf{a}, \mathbf{b}) \in g_{i j}\left(C_{0}\right)$ for some $i, j$, then $P(\mathbf{a})=P(\mathbf{b})$, as required.

Now, to see that this is true for all $(\mathbf{a}, \mathbf{b}) \in C_{1}$, note that by definition, $P(p(\mathbf{a}))=$ $P(\mathbf{a})$ for any permutation $p$ on $n-1$ letters, and therefore, if $P(\mathbf{a})=P(\mathbf{b})$ for $(\mathbf{a}, \mathbf{b}) \in C_{1}$, then the same holds for $(p(\mathbf{a}), p(\mathbf{b}))$. Finally, if $\left(\mathbf{a}_{1}, \mathbf{a}_{2}\right),\left(\mathbf{a}_{2}, \mathbf{a}_{3}\right) \in C_{1}$ have this property, then obviously $\left(\mathbf{a}_{1}, \mathbf{a}_{3}\right)$ also does. This completes the proof.

Theorem 6.5. If $E$ is a one-based theory generated by a balanced equation $(\mathbf{c}, \mathbf{d})$, then $E$ is definable in $\mathcal{L}(\mathrm{Com})$.

Proof. Let $\mathcal{M}=\mathcal{M}(\mathbf{c}, \mathbf{d})$ be the set of the theories generated by sets of equations of the form $(\mathbf{c}, p(\mathbf{d}))$. This set of theories is finite, and definable, by Theorem 5.4 Let $\mathcal{M}_{d}$ be the set of the theories $E \in \mathcal{M}$ such that, for each $T \in \mathcal{M}$, if $T \subseteq E$, then $T$ is individually definable in $\mathcal{L}(\mathrm{Com})$ (the theories in $\mathcal{M}_{d}$ may be said to be 
hereditarily definable with respect to $\mathcal{M}$ ). Since $\mathcal{M}_{d}$ is finite, it is definable. Our goal is to show that the set $\mathcal{M} \backslash \mathcal{M}_{d}$ of the remaining theories is empty.

Assume to the contrary, that it is not the case, and let $\mathcal{M}_{0}$ denote the set of minimal theories in the set $\mathcal{M} \backslash \mathcal{M}_{d}$. Then, $\mathcal{M}_{0}$ is definable and nonempty.

Let $E_{0} \in \mathcal{M}_{0}$. Then, by definition, each proper subtheory of $E_{0}$ in $\mathcal{M}$ is definable, and therefore $E_{0}$ has an equation $(\mathbf{c}, f(\mathbf{d}))$ such that $E(\mathbf{c}, f(\mathbf{d}))$ is not definable. It follows also that $E_{0}=E(\mathbf{c}, f(\mathbf{d}))$. In order to apply our lemmas directly, without loss of generality, we may assume that $E_{0}=E(\mathbf{c}, \mathbf{d})$.

Consider now the following formula:

$\Psi(E): E \in \mathcal{M}_{0}$, and for each pair $\mathbf{a}, \mathbf{b}$, such that $\mathbf{a}=p_{1} g_{i j}(\mathbf{c})$ and $\mathbf{b}=p_{2} g_{r s}(\mathbf{d})$ for some permutations $p_{1}, p_{2}$, and $P(\mathbf{a}) \neq P(\mathbf{b})$, no equation of the form $(\mathbf{a}, q(\mathbf{b}))$ is in $E$.

(Of course, it is assumed above that $1 \leq i, j, r, s \leq n$ with $i \neq j, r \neq s$, and we recall that the operation $P$ is defined with respect to $\Pi=\Pi(\mathbf{c}, \mathbf{d})$ equivalence.)

Since there are finitely many sequences of the form $\mathbf{a}=g_{i j}(\mathbf{c})$ and $\mathbf{b}=g_{r s}(\mathbf{d})$, $\Psi(E)$ can be expressed as a conjunction of " $E \in \mathcal{M}_{0}$ " and a finite conjunction of formulas of the form: "no equation of the form $(\mathbf{a}, q(\mathbf{b}))$ is in $E$ ". By assumption, and in view of Theorem 5.4 this can be expressed in the first-order language of $\mathcal{L}(\mathrm{Com})$. Obviously, by Lemma 6.4 $E(\mathbf{c}, \mathbf{d})$ satisfies this formula, and we show that this is actually the only theory satisfying it.

Assume that $\Psi(E)$ holds. Then $E \in \mathcal{M}_{0}$, and as we have already observed above, $E=E(\mathbf{c}, f(\mathbf{d}))$ for some permutation $f$. We show that $f \in G(\mathbf{c}, \mathbf{d})$.

Suppose that $f$ is not a member of $G(\mathbf{c}, \mathbf{d})$. Then there is $i \leq n$ such that $f(i)$ is not $\Pi$-equivalent to $i$. Since $n>2$, we may choose $r \leq n$ such that $r \neq i$ and $[i] \cup[r] \neq[f(i)] \cup[f(r)]$ (where the braces denote $\Pi$-equivalence classes). Indeed, if there is $r \in[i]$ with $r \neq i$, then such an $r$ is as required. Otherwise, since $n>2$, there is $r \neq i, f^{-1}(i)$. It follows that $f(r) \notin[i]$, and as $f(i) \notin[i]$, the claim follows. Consequently, if we put $(\mathbf{a}, \mathbf{b})=\left(g_{i r}(\mathbf{c}), g_{i r} f(\mathbf{d})\right)$, then $P(\mathbf{a}) \neq P(\mathbf{b})$. Finally, since $g_{i r} f(\mathbf{d})$ may be presented in the form $f_{1} g_{j s}(\mathbf{d})$, for some permutation $f_{1}$ on $n-1$ elements, by virtue of $\Psi(E),(\mathbf{a}, \mathbf{b}) \notin E$, which contradicts the fact that $(\mathbf{a}, \mathbf{b})$ is a direct consequence of $(\mathbf{c}, f(\mathbf{d}))$.

Thus, $f \in G(\mathbf{c}, \mathbf{d})$, and it follows, by Lemma 6.2 that $(\mathbf{c}, f(\mathbf{d})) \in E(\mathbf{c}, \mathbf{d})$. By $\Psi(E)$ and minimality of $E(\mathbf{c}, \mathbf{d}), E(\mathbf{c}, f(\mathbf{d}))=E(\mathbf{c}, \mathbf{d})$, as claimed.

It follows that $E(\mathbf{c}, \mathbf{d})$ is definable. Since, by assumption, each proper subtheory of $E(\mathbf{c}, \mathbf{d})$ in $\mathcal{M}$ is definable, we infer that $E(\mathbf{c}, \mathbf{d}) \in \mathcal{M}_{d}$, which is a contradiction. Hence, $\mathcal{M}_{d}=\mathcal{M}$, and the proof is completed.

Example 6.6. Consider the theory $E_{0}$ generated by the equation

$$
x^{2} y^{2} z^{4} t^{5}=x y^{3} z^{3} t^{6} .
$$

We have $\mathbf{c}=(2,2,4,5), \mathbf{d}=(1,3,3,6),(\mathbf{c}, \mathbf{d})$ is balanced, and $E_{0}=E(\mathbf{c}, \mathbf{d})$. The relation $\Pi=\Pi(\mathbf{c}, \mathbf{d})$ has two blocks, $\{1,2,3\}$ and $\{4\}$. Let $\mathbf{a}=p_{1} g_{i j}(\mathbf{c})$ and $\mathbf{b}=p_{2} g_{r s}(\mathbf{d})$ for some permutations $p_{1}, p_{2}$. Then, $P(\mathbf{a})=P(\mathbf{b})$ holds either if both the sides equal $\{1,2,3\}$ or if both the sides equal $\{1,2,3,4\}$. The former means that $\{i, j\},\{r, s\} \subseteq\{1,2,3\}$, and the latter means that $4 \in\{i, j\} \cap\{r, s\}$.

Let $\Psi^{\prime}(E)$ denote the formula obtained from $\Psi(E)$ in the proof above by deleting the conjunct $E \in \mathcal{M}_{0}$. It follows that if $(\mathbf{c}, p(\mathbf{d})) \in E$ and $\Psi^{\prime}(E)$ holds, then $p$ fixes 4. Yet, all equations $(\mathbf{c}, p(\mathbf{d}))$ with this property are equivalent to each other. Consequently, the formula $\Psi^{\prime}(E)$ defines $E_{0}$ in this case. 
Combining the facts that each equational theory in $\mathcal{L}(\mathrm{Com})$ is finitely axiomatizable (16]), and that each theory generated by a finite number of equations is a join of one-based theories, we obtain the following.

Corollary 6.7. If a theory $E$ is generated by a set of equations $\Sigma$ such that each equation in $\Sigma$ is either irregular, or with comparable nonequivalent sequences, or balanced, then $E$ is definable in $\mathcal{L}(C o m)$.

\section{Automorphism Group}

We look now at equations in two variables. In this case, it is not easy to distinguish between the consequences of such an equation and its companion obtained by transposition of variables on one of the sides. In fact, our attempts to do so have led to a counterexample.

In this section we construct a nontrivial automorphism on $\mathcal{L}(\mathrm{Com})$, exchanging certain one-based theories generated by equations in two variables.

The idea is as follows. Let $e_{1}$ and $e_{2}$ be two equations. For a theory $E \in \mathcal{L}(\mathrm{Com})$ we define $\phi(E)$ to be the set of equations such that for every equation $e$, the formula $e \in E \leftrightarrow e \in \phi(E)$ fails if and only if $e \in\left\{e_{1}, e_{2}\right\}$ and $E \cap\left\{e_{1}, e_{2}\right\}$ is a one-element set. Thus, we can say that $\phi(E)$ is obtained from $E$ by exchanging $e_{1}$ and $e_{2}$. Generally, $\phi(E)$ need not be a theory. Yet, if it happens to be a theory for every $E \in \mathcal{L}(\mathrm{Com})$, then $\phi$ is an automorphism of $\mathcal{L}(\mathrm{Com})$.

Indeed, it is a routine exercise to check that, in general,

$$
\phi\left(E_{1} \cap E_{2}\right)=\phi\left(E_{1}\right) \cap \phi\left(E_{2}\right) .
$$

A slightly more involved argument yields also the following fact.

Lemma 7.1. If $\phi(E)$ is a theory for every $E \in \mathcal{L}(C o m)$, then

$$
\phi\left(E_{1} \vee E_{2}\right)=\phi\left(E_{1}\right) \vee \phi\left(E_{2}\right),
$$

for all $E_{1}, E_{2} \in \mathcal{L}(C o m)$.

Proof. By assumption, $\phi\left(E_{1} \vee E_{2}\right)$ is a theory. It is easy to see that it contains both $\phi\left(E_{1}\right)$ and $\phi\left(E_{2}\right)$. Hence, $\phi\left(E_{1} \vee E_{2}\right) \supseteq \phi\left(E_{1}\right) \vee \phi\left(E_{2}\right)$.

For the converse inclusion, observe that, in turn, $\phi\left(\phi\left(E_{1}\right) \vee \phi\left(E_{2}\right)\right)$ contains both $E_{1}$ and $E_{2}$. This, by assumption, is also a theory, and therefore $\phi\left(\phi\left(E_{1}\right) \vee \phi\left(E_{2}\right)\right) \supseteq$ $E_{1} \vee E_{2}$. Using the fact that $\phi(\phi(E))=E$, we obtain $\phi\left(E_{1}\right) \vee \phi\left(E_{2}\right) \supseteq \phi\left(E_{1} \vee E_{2}\right)$, completing the proof.

It is not easy to find a nontrivial pair of equations satisfying the assumption of the lemma above. The first example found by the author was the pair of equations

$$
x^{3} y^{4}=x y^{5} \text { and } x^{4} y^{3}=x y^{5} .
$$

If $E$ contains both the equations, or none of them, then obviously $\phi(E)$ is a theory. We need to prove that if a theory $E$ contains only one of these equations, then after exchanging it with another one, we still have a theory. What makes this statement true in this particular case is that the filter of such a theory must be relatively large, while the remainder is relatively small, and there is no room for consequences that can distinguish one equation from another.

Ralph McKenzie has observed that also each pair of the equations

$$
x^{k} y^{k+1}=x y^{k+2} \text { and } x^{k+1} y^{k}=x y^{k+2}
$$


for any odd $k \geq 3$ has all the properties of the first example we need. This led to the surprising conclusion that the automorphism group of $\mathcal{L}(\mathrm{Com})$ embedded a Boolean group of continuum order. The proof below is a modification of the original proof to cover the general case.

Lemma 7.2. If $E=E(J, m, r, \pi)$ is a theory in $\mathcal{L}(C o m)$ containing exactly one of the equations of $(*)$ for a fixed odd $k \geq 3$, then the following conditions hold:

(i) $(k+3),(1, k, k+1),(1,1, k+2) \in J$.

(ii) If $(\mathbf{a}, \mathbf{b}) \in \pi, \mathbf{a} \neq \mathbf{b}$, and $\mathbf{b}=(1, k+2)$, then either $\mathbf{a}=(k, k+1)$ or $\mathbf{a}=(k+1, k)$.

(iii) If $(\mathbf{a}, \mathbf{b}) \in \pi, \mathbf{a} \neq \mathbf{b}$, and $f$ is one of the operations $g_{i j}$ or $h_{1}$, then $f(\mathbf{a})$ is different from $(1, k+2)$ and $(k+2,1)$.

Proof. Our assumption is that either $e_{1}=((k, k+1),(1, k+2))$ or $e_{2}=$ $((k+1, k),(1, k+2))$ is in $E$, and exactly one of these cases holds. These cases are not quite symmetric, but we try to treat them simultaneously.

First of all, note that, in any case, $m \leq 1$ and $r=1($ note that $\operatorname{gcd}(k, 2)=1$, since $k$ is odd). By definition of $E(J, m, r, \pi)$, either $e_{i} \in \pi$ for some $i=1$, 2 , or both $(k, k+1),(1, k+2) \in J$. In the latter case, by the same definition, both $e_{1}, e_{2} \in E$, contradicting the assumption. Hence, $e_{i} \in \pi$ is the case.

By $(\pi 3),(k+1, k, 1),(k+2,1,1) \in J$, and by $(\pi 4),(k+3) \in J$, proving (i).

Suppose that $(\mathbf{a}, \mathbf{b}) \in \pi, \mathbf{a} \neq \mathbf{b}$, and $\mathbf{b}=(1, k+2)$. To fix attention, suppose that $e_{1} \in \pi$, i.e., $(k, k+1),(1, k+2)$ are in a block of $\pi$, and suppose that $\mathbf{a}=(\alpha, \beta)$ is another element in this block. Recall that sequences in a block are incomparable or equivalent. If a were equivalent to any of $(k, k+1),(1, k+2)$, a would be $(k+1, k)$ or $(k+2,1)$. In any of these cases, we would have in consequence (using $(\pi 2)$ ) that both $e_{1}, e_{2} \in \pi$, which contradicts the assumption. Hence, $\mathbf{a}$ is incomparable with $(k, k+1)$ and $(1, k+2)$. It follows that both $\alpha$ and $\beta$ are different from any of $k, k+1, k+2,1$. Also, since $(k+3) \in J$, both $\alpha, \beta<k+3$. Consequently, $1<\alpha, \beta<k$, which implies that $\mathbf{a}<(k, k+1)$, again contradicting the assumption. It follows that there are no other elements in a block containing $(k, k+1),(1, k+2)$, proving (ii) in this case. Exactly the same argument works for the case $e_{2} \in \pi$.

For (iii), let $(\mathbf{a}, \mathbf{b}) \in \pi$. Obviously, for $f=h_{1}$, since $\mathbf{a}$ has length at least $2, f(\mathbf{a})$ is different from $(1, k+2)$ and $(k+2,1)$. So, we may assume that $f$ is one of the $g_{i j}$. Assume also, to the contrary, that $f(\mathbf{a})=(1, k+2)($ for $(k+2,1)$ the argument is the same). Then, by (ii), since $(1, k+2) \notin J, f(\mathbf{b})=(k, k+1)$ or $(k+1, k)$. Assume that $f(\mathbf{b})=(k, k+1)$ (again, for $(k+1, k)$ the argument is the same, since it does not depend on permutation of sequences).

Then $\mathbf{a}, \mathbf{b}$ must be three-element sequences with $g_{i j}(\mathbf{a})=(1, k+2)$ and $g_{i j}(\mathbf{b})=$ $(k, k+1)$. It follows that, up to permutation, $\mathbf{a}=(1, k+2-r, r)$, and $\mathbf{b}=$ $(k-s, s, k+1)$ or $\mathbf{b}=(k, k+1-t, t)$ for some $r, s, t \geq 1$ with $r<k+2, s<k$, and $t<k+1$.

Now, observe that while $g_{i j}(\mathbf{a})$ does not belong to $J$ for any $i, j$ (since in any case the resulting two-element sequence is less than or equal to $(1, k+2)$ or $(k, k+1))$, this is not the case with $g_{i j}(\mathbf{b})$. Indeed, if $\mathbf{b}=(k-s, s, k+1)$, then $g_{13}(\mathbf{b})$ or $g_{23}(\mathbf{b})$ is greater then $(k+3)$, and if $\mathbf{b}=(k, k+1-t, t)$, then $g_{13}(\mathbf{b})$ or $g_{12}(\mathbf{b})$ is greater then $(k+3)$. Since $(\mathbf{a}, \mathbf{b}) \in \pi$, this leads to a contradiction with condition $(\pi 4)$. 
Now, given an odd $k \geq 3$, let $\phi_{k}(E)$ denote $\phi(E)$ applied to the equations $(*)$, and assume that exactly one of these equations is in $E$. Then $E$ is nontrivial, and $E=E(J, m, r, \pi)$ for some $J, m, r$, and $\pi$. We prove that $\phi_{k}(E)$ is a theory of the form $E\left(J, m, r, \pi^{\prime}\right)$ for some remainder $\pi^{\prime}$ of type $(J, m, r)$.

By Lemma 7.2, either $((k, k+1),(1, k+2))$ or $((k+1, k),(1, k+2))$ is in $\pi$, and moreover, the block containing $(1, k+2)$ consists of only two elements. By $(\pi 2)$, also the block containing $(k+2,1)$ consists of exactly two elements, and in any case, these remaining elements are $(k, k+1)$ and $(k+1, k)$, placed in one of the two possible ways. Hence, it is enough to prove that if $\phi_{k}(\pi)$ denotes $\pi$ after exchanging $(k, k+1)$ and $(k+1, k)$ in blocks, then $\phi_{k}(\pi)$ is a remainder of type $(J, m, r)$.

Obviously, $\phi_{k}(\pi)$ is still an equivalence relation on $\Gamma_{+} \backslash J$, every block in $\phi(\pi)$ contains the sequences of the same length, and conditions $(\pi 1, \pi 2)$ trivially hold. For condition $(\pi 3)$, note that exchanging $(k, k+1)$ and $(k+1, k)$ in two-element blocks changes nothing, and $(\pi 3)$, still holds. Finally, for $(\pi 4)$, from Lemma 7.2(iii) we see that nontrivial consequences of any $(\mathbf{a}, \mathbf{b}) \in \pi$ do not fall into the blocks with $(k+2,1)$. Hence, $(\pi 4)$ holds for $\phi_{k}(\pi)$, as well.

Using Lemma 7.1 we infer that $\phi_{k}(E)$ is an automorphism, which has the following important consequence.

Theorem 7.3. There are theories in $\mathcal{L}(\mathrm{Com})$ that are not definable in the firstorder language.

Since we now have a denumerable list of automorphism, we may infer, in addition, that the group of automorphisms is infinite. Yet, this may be still strengthened. Namely, let $S$ be a subset of the set of odd integers $k \geq 3$. We define $\phi_{S}(E)$ to be the result of applying $\phi_{k}$ to $E$ for all $k \in S$. This is well-defined, since the results of applying different $\phi_{k}$ are independent from each other. This is also why the proof of Lemma 7.1 and the remarks preceding it apply to $\phi_{S}(E)$ as well. In this way we obtain the result established during McKenzie's seminar at Vanderbilt.

Theorem 7.4. The automorphism group of $\mathcal{L}(\mathrm{Com})$ contains a Boolean group of continuum order, as a subgroup.

An interesting, still open problem is how complex the automorphism group of $\mathcal{L}(\mathrm{Com})$ actually is. Are there any automorphisms of order greater than 2? Are there automorphisms of some other type than those obtained by exchanging equations?

\section{ACKNOWLEDGMENT}

The results of this paper were presented in a series of talks during Ralph McKenzie's seminar at Vanderbilt in 2002. I am very much indebted to the attendants of this seminar, especially, J. Ježek, P. Jipsen, P. Marković, and Ralph McKenzie himself, for their interest in the topic and many valuable remarks that helped me to improve the paper considerably. Also, I would like to take this opportunity to thank Ralph and Kathy McKenzie for their hospitality and friendship.

\section{REFERENCES}

[1] M. Grech, Irreducible varieties of commutative semigroups, J. Algebra, to appear.

[2] M. Grech, A. Kisielewicz, Covering relation for equational theories of commutative semigroups, J. Algebra 232 (2000), 493-506. MR 2002g:20102 
[3] P. A. Grillet, Fully invariant congruences on free commutative semigroups, Acta Sci. Math. (Szeged) 67 (2001), 571-600. MR 2003d:20093

[4] A. A. Iskander, Coverings in the lattice of varieties, in Contributions to universal algebra, pp. 189-203., Colloq. Math. Soc. Janos Bolyai, Vol. 17, North-Holland, Amsterdam, 1977. MR 58:16462

[5] A. A. Iskander, Definability in the lattice of ring varieties, Pacific J. Math. 76 (1978), 61-67. MR 58:448

[6] J. Ježek, The lattice of equational theories I: Modular elements, Czech. Math. J. 31 (1981), 127-152. MR 84e:08007a

[7] - The lattice of equational theories II: The lattice of full sets of terms, Czech. Math. J. 31 (1981), 127-152. MR 84e:08007b

[8] - The lattice of equational theories III: Definability and automorphisms, Czech. Math. J. 32 (1982), 129-164. MR 84e:08007c

[9] - The lattice of equational theories IV: Equational theories of finite algebras, Czech. Math. J. 36 (1986), 331-341. MR 87g:08015

[10] J. Ježek, R. McKenzie, Definability in the lattice of equational theories of semigroups, Semigroup Forum 46 (1993), 199-245. MR 94a:03052

[11] A. Kisielewicz, Varieties of commutative semigroups, Trans. Amer. Math. Soc. 342 (1994), 275-306. MR 94j:20065

[12] — , All pseudovarieties of commutative semigroups, in Semigroups with applications (Oberwolfach, 1991), (J. M. Howie et al. eds), World Scientific, Singapore, 1992, pp. 7889. MR 93k:20086

[13] — , Unification in commutative semigroups, J. Algebra 200 (1998), 246-257. MR 99g:20107

[14] R. McKenzie, Definability in lattices of equational theories, Annals of Math. Logic 3 (1971), 197-237. MR 43:6069

[15] E. Nelson, The lattices of equational classes of commutative semigroups, Canad. J. Math. 23 (1971), 875-895. MR 44:5395

[16] P. Perkins, Bases for equational theories of semigroups, J. Algebra 11 (1969), 298-314. MR 38:2232

[17] A. Tarski, Equational logic and equational theories of algebras, Contributions to Mathematical Logic (Hannover, 1966), 275-288, North-Holland, Amsterdam, 1968. MR 38:5692

[18] Vernikov, B. M., Definable varieties of associative rings, Mat. Issled. No. 90, Algebry i Koltsa (1986), 41-47, (Russian). MR 88e:08012

Institute of Mathematics, University of Wroceaw, Pl. Grunwaldzki 2/4, 50-384 Wroceaw, Poland

E-mail address: kisiel@math.uni.wroc.pl 